\title{
Synthesis of 1,5-Bifunctional Organolithium Reagents by a Double Directed ortho- Metalation: Direct Transformation of Esters into 1,8-Dimethoxy-Acridinium Salts
}

\author{
Christian Fischer, Christof Sparr* \\ ${ }^{a}$ University of Basel, Department of Chemistry, St. Johanns-Ring 19, 4059 Basel, Switzerland
}

ARTICLE INFO

ABSTRACT

Article history:

Received

Received in revised form

Accepted

Available online

Keywords:

Carboxylic Acid Ester;

Fluorophores;

Organometallic Reagents;

Directed ortho-Metalation;

Acridinium Salts;

Photoredox Catalysis

\begin{abstract}
The impact of electronic and steric factors on the selectivity of the electrophilic aromatic substitution amounts to several limitations in accessing specific substitution patterns. Nucleophiles generated by directed metalation represent an effective alternative for the preparation of various distinctly substituted arenes and heterocyclic scaffolds to overcome these restraints. Herein, we report the direct synthesis of specifically substituted heterocyclic fluorophores from esters by the addition of 1,5-bifunctional organometallic reagents from a double directed ortho-metalation $(\mathrm{dD} o \mathrm{M})$. Bis(3-methoxylphenyl)amines were efficiently dilithiated und employed for the synthesis of 1,8-dimethoxy-acridinium salts with distinct photophysical and electrochemical properties. The individual reduction potentials, the watersolubility and the brightness of these new dyes promise different applications in catalysis, imaging and materials science.
\end{abstract}

2018 Elsevier Ltd. All rights reserved.

\section{Introduction}

The possibility to adjust photophysical and electrochemical properties of luminescent metal complexes by variation of pyridine-based ligands crucially contributed to the advancement of photocatalysis in recent years. ${ }^{1}$ In analogy, organic fluorophores would allow complementary and sustainable catalytic processes, ${ }^{2}$ but are currently not accessible in similar variety despite substantial progress. As heterocyclic fluorophores are usually prepared by electrophilic aromatic substitution methods, their substitution pattern is typically governed by electronic and steric factors that limit the dye diversity. ${ }^{3}$ Recently, we have investigated an alternative approach by the use of 1,5-bifunctional organometallic reagents from halogen-metal exchange reactions for the double addition to carboxylic acid esters. ${ }^{4}$ Upon dehydration, this transformation enables the direct conversion of various esters into valuable heterocyclic fluorophores with high functional group tolerance. ${ }^{5}$ However, the use of dimetallic reagents not only allows the direct transformation of stable and readily available carboxylic acid esters, ${ }^{6}$ but would also provide a means to prepare heterocyclic compounds with a substitution pattern different to electrophilic aromatic substitution products of the same bis(3methoxyphenyl)-amine precursors 1 (Figure 1 left). ${ }^{7}$ More specifically, reagents prepared by a double directed ortho-metala-

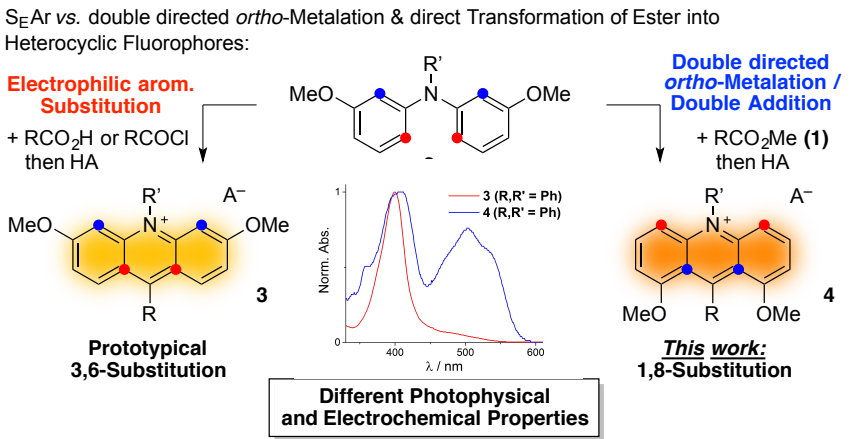

Figure 1. Varying substitution pattern from a common tertiary amine: electrophilic aromatic substitution and the double directed ortho-metalation for the direct transformation of esters into 1,8dimethoxy acridinium salts with different photophysical and electrochemical properties.

tion $(\mathrm{dD} o \mathrm{M})$ of bis(3-methoxyphenyl)-amines 1 would give rise to uncommonly substituted 1,8-dimethoxy acridinium salts 4 , expected to exhibit distinct physical and chemical properties. ${ }^{8}$ The resulting 1,8-substitution of the products from the double directed ortho-metalated reagent therefore provides a specific peri-relationship of the methoxy groups with the ester residue (R, Figure 1 right).

\footnotetext{
$\$$ Dedicated to the memory of Sir Derek H. R. Barton FRS FRSE.

*Corresponding author. Tel.: +41 6120711 10; e-mail: christof.sparr@unibas.ch
} 


\section{Results and Discussion}

\subsection{Synthesis of Tertiary Amine Precursors}

To investigate the feasibility and applicability of this double directed ortho-metalation strategy, we anticipated a synthesis of tertiary bis(3-methoxyphenyl)-amines $\mathbf{1}$ from simple, commercially available building blocks. For further diversification, we prepared 3-bromo-5-methoxy- $N, N$-dimethylaniline (7) in four steps, involving a bromination of 1,3dinitrobenzene using $N$-bromosuccinimide followed by nucleophilic aromatic substitution with sodium methoxide, reduction and dimethylation by reductive amination of the aniline (Scheme 1). ${ }^{9}$ In the reductive amination step, the yield was improved by limiting the contact time of the aniline with formaldehyde in the acidic medium by the portionwise addition of a suspension of $\mathrm{NaBH}_{4}$ and aniline in THF to the acidic aqueous formaldehyde solution. ${ }^{10}$

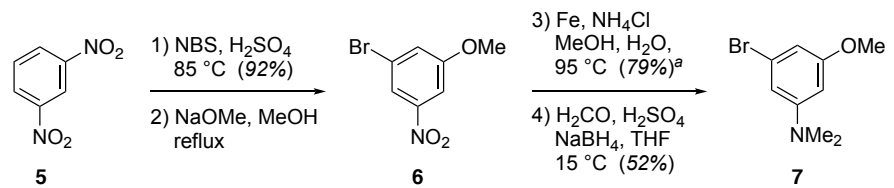

Scheme 1. Synthesis of arylbromide 7. ( ${ }^{a}$ Yield over two steps.)

Several tertiary amines were synthesized within one or two steps with aryl halides and anilines (Scheme 2), whereas the methoxy-residues were kept consistent as directed metalation group (DMG). Buchwald-Hartwig amination reactions allowed a high-yielding synthesis of secondary and tertiary amines by using $\mathrm{Pd}_{2}(\mathrm{dba})_{3}$ with appropriate ligands (1,1'-bis(diphenylphosphino)ferrocene (dppf) for secondary amines and 2dicyclohexylphosphino-2', $6^{\prime}$-diisopropoxybiphenyl (RuPhos) for tertiary amines). Moreover, double amination gave direct access to the tertiary amine $1 \boldsymbol{a}$ and $\boldsymbol{l c}$ in $84 \%$ and $47 \%$ yield, respectively. Alternatively, the secondary amine intermediates were either methylated with iodomethane in the presence of sodium hydride ( $\mathbf{l d}, 81 \%$ yield) or arylated to provide triarylamine $\mathbf{1 b}$ in $98 \%$ yield.

a)

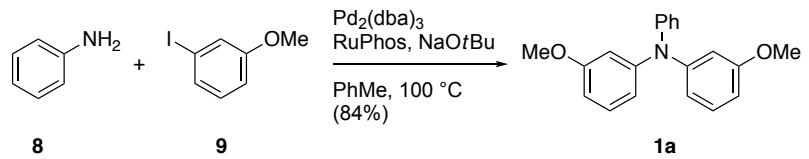

b)

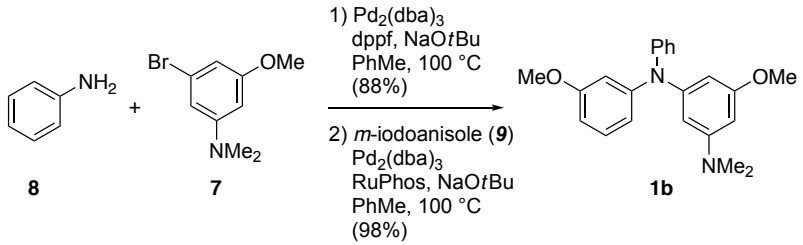

c)
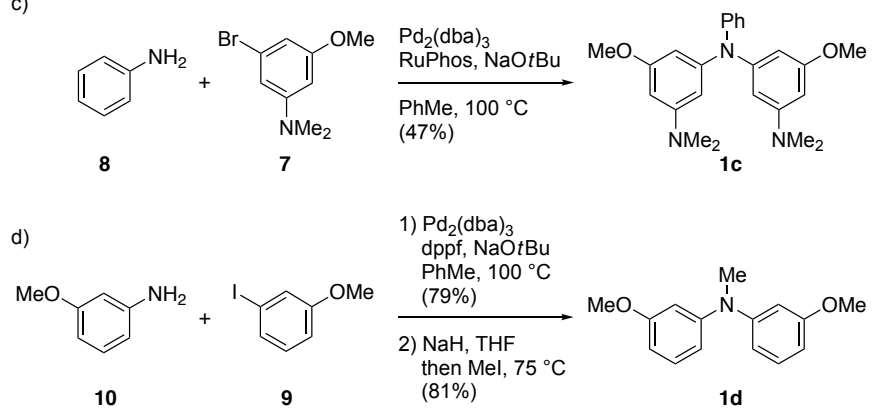

Scheme 2. Synthesis of various acridinium backbones $\mathbf{1 a - d}$.
With the tertiary amine precurscors $\mathbf{1 a}-\boldsymbol{d}$ in hand, the reagent synthesis by a double directed ortho-metalation and the subsequent addition to carboxylic acid esters was investigated.

\subsection{Synthesis of 1,5-Bifunctional Organolithium Reagents by a Double Directed ortho-Metalation}

Owing to the expeditious one-step synthesis of amine $1 a$, the optimization parameters of the double directed ortho-metalation were investigated by the transformation of methyl benzoate $(2 a)$ into acridinium salt $\mathbf{4 a}$ (Table 1).

Mainly the time for the directed ortho-metalation and the temperature for the direct ester transformation were found crucial and were examined in detail. As no metalation was observed at room temperature, the double directed ortho-metalation was performed at $65{ }^{\circ} \mathrm{C}$ throughout the optimization experiments. Furthermore, the reaction work-up by treatment with aq. $\mathrm{HBr}$ was kept consistent to uniformly form the corresponding acridinium bromide salt. Initially, three hours of metalation, followed by the addition of methyl benzoate at $-20{ }^{\circ} \mathrm{C}$ and subsequent reaction at $65{ }^{\circ} \mathrm{C}$ over 12 hours followed by aq. $\mathrm{HBr}$ treatment led to the formation of $28 \%$ acridinium salt $4 a$ (Table 1, entry 1). An attempt to transmetalate lithium to magnesium under the same conditions to attenuate the reactivity of the 1,5-bifunctional organometallic reagent did not improve the yield (entry 2). However, by extending the metalation period to 6 hours and allowing the ester to react at either $65{ }^{\circ} \mathrm{C}$ or room temperature resulted in an increased product formation $(40 \%$ and $43 \%$ yield, entries $3 \& 4)$. Addition of $\mathrm{Et}_{2} \mathrm{O}(\sim 9 \mathrm{v} / \mathrm{v} \%)$, to activate $n \mathrm{BuLi}$ to promote the lithiation ${ }^{11}$ did not significantly change the reactivity (entry 5 ). Extending the metalation to 12 hours decreased product formation (32\%, entry 6) and by an alternative lithiation with TMPLi (lithium tetramethylpiperidide) with or without transmetalation using a $\mathrm{MgCl}_{2} \cdot \mathrm{LiCl}$ solution, the formation of acridinium bromide salt $\mathbf{4 a}$ was not observed. ${ }^{12}$

Table 1. Optimization of the double directed ortho-metalation (DoM) and in situ addition of carboxylic acid esters (2). ${ }^{13}$

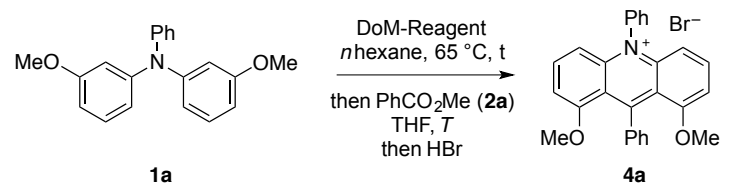

\begin{tabular}{ccccc}
\hline Entry & DoM-Reagent & $\mathrm{t}$ & $T$ & Isolated Yield \\
\hline 1 & $n \mathrm{BuLi}^{\mathrm{a}}$ & $3 \mathrm{~h}$ & $65^{\circ} \mathrm{C}$ & $28 \%$ \\
2 & $n \mathrm{BuLi}^{\mathrm{a}}$ & $3 \mathrm{~h}$ & $65^{\circ} \mathrm{C}$ & $25 \%$ \\
3 & $n \mathrm{BuLi}$ & $6 \mathrm{~h}$ & $65^{\circ} \mathrm{C}$ & $40 \%$ \\
4 & $n \mathrm{BuLi}$ & $6 \mathrm{~h}$ & $\mathrm{RT}$ & $\mathbf{4 3 \%}$ \\
5 & $n \mathrm{BuLi}, \mathrm{Et} \mathrm{O}^{\mathrm{b}}$ & $6 \mathrm{~h}$ & $\mathrm{RT}$ & $39 \%$ \\
6 & $n \mathrm{BuLi}^{\circ}$ & $12 \mathrm{~h}$ & $65^{\circ} \mathrm{C}$ & $32 \%$ \\
7 & $\mathrm{TMPLi}^{\mathrm{c}}$ & $1 \mathrm{~h}$ & $\mathrm{RT}$ & - \\
\hline
\end{tabular}

aAfter DoM, mixture was treated with $\mathrm{MgCl}_{2} \cdot \mathrm{LiCl}$ solution (in THF, $\left.0.50 \mathrm{molL}^{-1}, 0.64 \mathrm{~mL}\right)$ and then ester $2(100 \mu \mathrm{mol})$ in THF $(0.60 \mathrm{~mL})$ at $-20{ }^{\circ} \mathrm{C}$; ${ }^{b}$ Metalation in $n$ hexane $(2.0 \mathrm{~mL})$ and $\mathrm{Et}_{2} \mathrm{O}(0.20 \mathrm{~mL})$; ${ }^{\mathrm{c}}$ Metalation performed in $\mathrm{THF}(0.50 \mathrm{~mL})$ at $-40{ }^{\circ} \mathrm{C}$, followed by addition of Ester $1(100 \mu \mathrm{mol})$ in THF $(0.60 \mathrm{~mL})$ and warmed to RT. 


\subsection{Direct Synthesis of 1,8-substituted Acridinium Dyes}

With the optimized conditions for the double directed orthometalation and ester to acridinium dye synthesis, we evaluated the dehydrative work-up with aqueous $\mathrm{HBF}_{4}$ instead of hydrobromic acid, giving access to acridinium $\mathrm{BF}_{4}^{-}$salt $4 \boldsymbol{a}$ in similar yields (Table 2, entry 1). Furthermore, the scope of the double directed ortho-metalation and addition to methyl benzoate was studied with different tertiary amine precursors $\mathbf{1 a} \boldsymbol{d}$. The presence of one or two $\mathrm{NMe}_{2}$ moieties retards regioselective double lithiation. Nevertheless, acridinium dyes $4 b$ and $4 c$ could be synthesized in $26 \%$ and $35 \%$ yield, respectively (entry $2 \& 3$ ). By using amine $1 \boldsymbol{d}$ and methyl benzoate, a higher efficiency in the transformation into acridinium bromide salt $\mathbf{4} \boldsymbol{d}$ was observed ( $49 \%$ yield, entry 4$)$. Whereas treatment of the reaction mixture with strong aqueous acids such as hydrobromic acid or $\mathrm{HBF}_{4}$ promoted the elimination, treatment with ammonium chloride gave access to the intermediary 9,10-dihydroacridin-9-ol $4 \boldsymbol{e}$ in $43 \%$. The reactivity of the $N$-aryl- $N$-methylaniline $1 a$ was further investigated by varying the ester substrates. Both esters with an electron-withdrawing fluorine substituent (methyl 4fluorobenzoate) or an electron-donating methoxy group (methyl $p$-anisate) yielded acridinium salts $\boldsymbol{4} \boldsymbol{f}$ and $\boldsymbol{4 g}$ in $41 \%$ and $39 \%$ respectively (entries $6 \& 7$ ). The sterically more demanding methyl 1-naphthoate was transformed into acridinium bromide salt $4 \boldsymbol{d}$ in a yield of $57 \%$.

\subsection{Photophysical \& Electrochemical Properties}

As the prepared acridinium salts 4 are characterized by an unusual substitution pattern, we studied the photophysical and electrochemical properties to assess their utility as fluorophores in imaging or photochemistry.

We therefore measured cyclic voltammetry and determined the absorption wavelength, molar attenuation coefficient, fluorescence emission wavelength and the fluorescent lifetime as well as the excitation energy $E_{0,0}$ to determine the reduction potential in the ground and excited state. In comparison to prototypical acridinium salts, both the absorption and emission of acridinium dyes $\mathbf{4} \boldsymbol{a}-\boldsymbol{h}$ are significantly red-shifted, exhibiting an average Stokes shift of larger than $70 \mathrm{~nm}$ (Table 3).
Table 2. Direct Transformation of Esters into 1,8-substituted acridinium dyes $^{\mathrm{a}, \mathrm{b}}$ and 9,10-dihydroacridin-9-ol. ${ }^{\mathrm{c}}$
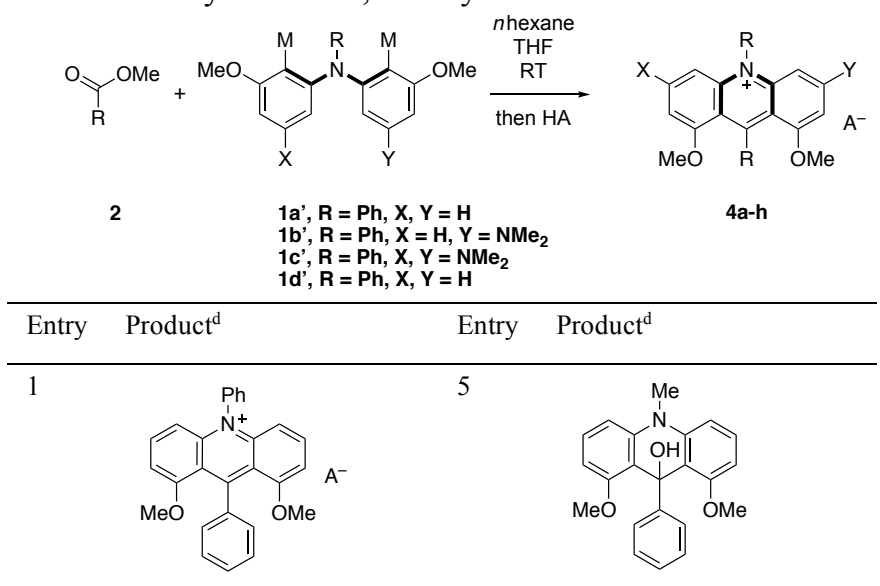

4a, $\mathrm{Br}^{-}, 43 \% ; \mathrm{BF}_{4}^{-}, 44 \%{ }^{\mathrm{b}}$

2

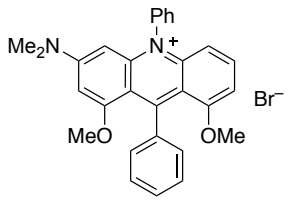

4b, $26 \%$

3

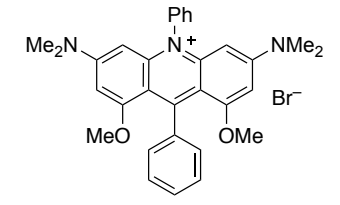

4c, $35 \%$

4

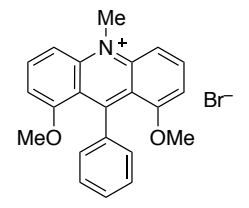

4d, $49 \%$
$4 \mathrm{e}, 43 \%^{\mathrm{c}}$

6
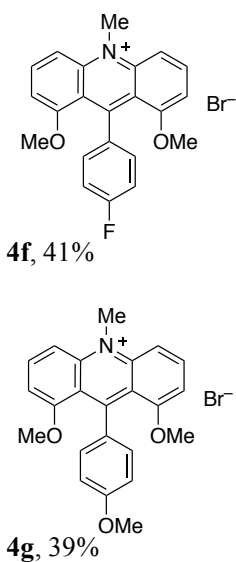

8

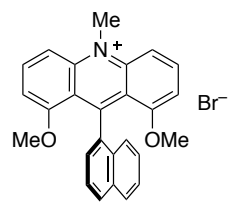

4h, $57 \%$
${ }^{\mathrm{a}}$ Reactions performed with $\mathbf{1}(100 \mu \mathrm{mol})$ in THF $(0.60 \mathrm{~mL})$ and $2 \boldsymbol{a}^{\prime} \boldsymbol{- \boldsymbol { d }}$ ' $(\mathrm{M}=$ $\mathrm{Li} ; 160 \mu \mathrm{mol})$ for $12-14 \mathrm{~h}$ at RT followed by aqueous work-up $\left(8.8 \mathrm{molL}^{-1}\right.$, $\mathrm{HBr}$ ); ${ }^{b}$ Aqueous work-up using aq. $\mathrm{HBF}_{4}, 50 \%$; ${ }^{\mathrm{c}}$ Aqueous work-up using aq. sat. $\mathrm{NH}_{4} \mathrm{Cl} ;{ }^{\text {d}}$ Yields of isolated products.

Table 3. Photophysical and Electrochemical Properties of Acridinium Dyes $\mathbf{4 a}-\mathbf{4 h}$.

\begin{tabular}{|c|c|c|c|c|c|c|c|c|c|}
\hline Entry & Compound & $\lambda_{\text {abs,max }}{ }^{a}$ & $\varepsilon_{\max }\left[\mathrm{L} \cdot \mathrm{cm} \cdot \mathrm{mol}^{-1}\right]^{\mathrm{a}}$ & $\lambda_{\mathrm{em}, \max }{ }^{\mathrm{a}, \mathrm{b}}$ & Stokes Shift & $\mathrm{E}_{0,0}{ }^{\mathrm{a}}$ & $\mathrm{E}_{1 / 2}\left(\mathrm{P} / \mathrm{P}^{-}\right)^{\mathrm{c}}$ & $\mathrm{E}_{1 / 2}\left(\mathrm{P}^{*} / \mathrm{P}^{-}\right)^{\mathrm{b}}$ & $<\tau_{\mathrm{F}}>^{\mathrm{a}}$ \\
\hline 1 & $4 a-B r^{-}$ & $503 \mathrm{~nm}$ & $4.4 \cdot 10^{3}$ & $595 \mathrm{~nm}$ & $92 \mathrm{~nm}$ & $2.23 \mathrm{eV}$ & $-0.47 \mathrm{~V}$ & $+1.76 \mathrm{~V}$ & $3.1 \mathrm{~ns}$ \\
\hline 2 & $4 a-B_{4}^{-}$ & $503 \mathrm{~nm}$ & $3.2 \cdot 10^{3}$ & $596 \mathrm{~nm}$ & $93 \mathrm{~nm}$ & $2.23 \mathrm{eV}$ & $-0.49 \mathrm{~V}$ & $+1.74 \mathrm{~V}$ & $3.4 \mathrm{~ns}$ \\
\hline 3 & $4 b$ & $501 \mathrm{~nm}$ & $8.6 \cdot 10^{3}$ & $584 \mathrm{~nm}$ & $83 \mathrm{~nm}$ & $2.25 \mathrm{eV}$ & $-0.94 \mathrm{~V}$ & $+1.31 \mathrm{~V}$ & $4.7 \mathrm{~ns}$ \\
\hline 4 & $4 c$ & $498 \mathrm{~nm}$ & $4.0 \cdot 10^{4}$ & $540 \mathrm{~nm}$ & $42 \mathrm{~nm}$ & $2.40 \mathrm{eV}$ & $-1.19 \mathrm{~V}$ & $+1.21 \mathrm{~V}$ & $4.4 \mathrm{~ns}$ \\
\hline 5 & $4 d$ & $497 \mathrm{~nm}$ & $4.4 \cdot 10^{3}$ & $576 \mathrm{~nm}$ & $79 \mathrm{~nm}$ & $2.33 \mathrm{eV}$ & $-0.52 \mathrm{~V}$ & $+1.81 \mathrm{~V}$ & $2.7 \mathrm{~ns}$ \\
\hline 6 & $4 f$ & $497 \mathrm{~nm}$ & $3.9 \cdot 10^{3}$ & $579 \mathrm{~nm}$ & $82 \mathrm{~nm}$ & $2.31 \mathrm{eV}$ & $-0.51 \mathrm{~V}$ & $+1.80 \mathrm{~V}$ & $3.0 \mathrm{~ns}$ \\
\hline 7 & $4 \mathrm{~g}$ & $494 \mathrm{~nm}$ & $4.5 \cdot 10^{3}$ & $567 \mathrm{~nm}$ & $72 \mathrm{~nm}$ & $2.30 \mathrm{eV}$ & $-0.62 \mathrm{~V}$ & $+1.68 \mathrm{~V}$ & $5.9 \mathrm{~ns}$ \\
\hline 8 & $4 h$ & $497 \mathrm{~nm}$ & $5.0 \cdot 10^{3}$ & $531 \mathrm{~nm}$ & $34 \mathrm{~nm}$ & $2.39 \mathrm{eV}$ & $-0.51 \mathrm{~V}$ & $+1.88 \mathrm{~V}$ & $4.1 \mathrm{~ns}$ \\
\hline
\end{tabular}

a from a $15 \mu \mathrm{mol} \cdot \mathrm{L}^{-1}$ dye solution in acetonitrile. ${ }^{\text {b}}$ Excited $10 \mathrm{~nm}$ below $\lambda_{\text {max,abs. }}{ }^{\mathrm{c}}$ Measured in dry, degassed $0.1 \mathrm{~mol} \cdot \mathrm{L}^{-1} \mathrm{n}$ butylammonium hexafluorophosphate in acetonitrile against SCE. 
All absorption spectra of $\boldsymbol{4 a}$ - $\boldsymbol{h}$, except for $\left(\mathrm{NMe}_{2}\right)_{2}$-acridinium dye $4 c$, show two major signals corresponding to the absorptionsignal of the arene and acridinium moiety. Similar to Fukuzumi's 9-mesityl-10-methylacridinium $\left(\mathrm{MesMeAcr}^{+}\right)$, the 1,8substitution also promotes the perpendicular orientation of the two ring systems that limits their $\pi$-conjugation. ${ }^{14}$ Furthermore, $N$-methyl to $N$-phenyl substitution was found not to influence the molar attenuation coefficient of $4 \boldsymbol{a}-\mathrm{Br}^{-}, 4 \boldsymbol{b}, 4 \boldsymbol{c}$ and $4 \boldsymbol{d}$. However, the higher the number of dimethylamino groups, the higher the coefficient. The reduction potential remains independent of the anion as well as the arene moiety, except for $\boldsymbol{4 g}$ which is substituted by electron-rich anisole that decreases the groundstate reduction potential marginally. A higher number of dimethylamino groups at the acridinium of salts $4 b$ and $4 c$ furthermore decreases the reduction potential significantly, which is also reflected in the excited state reduction potential. Moreover, 1,8-dimethoxy substituted acridinium dyes possess excellent water solubility and fluorescence excited state lifetimes, which render these salts as promising photocatalysts.

\section{Conclusion}

A double directed ortho-metalation $(\mathrm{dD} o \mathrm{M})$ strategy giving access to reagents for the direct transformation of esters into 1,8dimethoxy-acridinium salts with distinct photophysical and electrochemical properties is described. Starting from an identical tertiary amine motif, this method allows diverging from the substitution pattern obtained by electrophilic aromatic substitution reactions. Synthetic strategies that provide a means to modulate the properties of organic fluorophores would render organophotoredox catalysis more generally applicable and enable novel applications in imaging, sensing or materials science. Due to the unique photophysical and electrochemical properties and the excellent water-solubility, the products described in this article are part of a patent application. ${ }^{15}$ Current studies on the application of the acridinium salts for photocatalysis will be reported in due course.

\section{Experimental}

\subsection{Bis(3-methoxyphenyl)-amine Precursors Syntheses}

\subsubsection{3-Bromo-5-methoxy-N,N-dimethylaniline (7)}

Prepared according to modified literature procedures: ${ }^{16 a, b}$ To a solution of 1,3-dinitrobenzene $(5)(20.2 \mathrm{~g}, 120 \mathrm{mmol})$ in conc. $\mathrm{H}_{2} \mathrm{SO}_{4}(95 \%, 0.24 \mathrm{~L})$ at $85{ }^{\circ} \mathrm{C}$ was added $N$-bromosuccinimide $(29.9 \mathrm{~g}, 168 \mathrm{mmol})$ portionwise over $1 \mathrm{~h}$. The reaction was continued to stirr for $1 \mathrm{~h}$ at $85^{\circ} \mathrm{C}$, cooled and poured into ice water. The precipitate was filtered and washed with aq. sat. $\mathrm{Na}_{2} \mathrm{SO}_{3}$ and water to $\mathrm{pH} 7$. The solid was dried in vacuo to give 1-bromo-3,5-dinitrobenzene as beige-yellow solid (27.3 g, 92\%): $\mathrm{R}_{f} 0.60\left(\mathrm{CH}_{2} \mathrm{Cl}_{2} 100 \%\right) ;{ }^{1} \mathrm{H}$ NMR $\left(500 \mathrm{MHz}, \mathrm{CDCl}_{3}\right) \delta=9.00$ $\left(1 \mathrm{H}, \mathrm{t},{ }^{4} J 2.0, \mathrm{C} 4 H\right), 8.71\left(2 \mathrm{H}, \mathrm{d},{ }^{4} J 2.0, \mathrm{C} 2 H\right) ;{ }^{13} \mathrm{C} \mathrm{NMR}(125$ $\left.\mathrm{MHz}, \mathrm{CDCl}_{3}\right) \quad \delta=148.9$ (C3), 132.1 (C2), 123.9 (C1), 117.7 $(C 4)$. Analytical data is in agreement with literature. ${ }^{16 a}$

To a solution of 1-bromo-3,5-dinitrobenzene (12.4 g, $50.0 \mathrm{mmol})$ in $\mathrm{MeOH}(0.50 \mathrm{~L})$ was added sodium methoxide $(21.6 \mathrm{~g}, 400 \mathrm{mmol})$ at $\mathrm{RT}$ and stirred $5 \mathrm{~h}$ at reflux. The mixture was adjusted to $\mathrm{pH} 6$ with aq. $\mathrm{HCl}\left(1 \mathrm{molL}^{-1}\right)$, filtered and the filtrate was concentrated in vacuo. The residue was filtered and washed with water to obtain 1-bromo-3-methoxy-5nitrobenzene (6), which was directly used in the next step. $\mathrm{R}_{f} 0.83\left(\mathrm{CH}_{2} \mathrm{Cl}_{2} 100 \%\right) ;{ }^{1} \mathrm{H} \mathrm{NMR}\left(500 \mathrm{MHz}, \mathrm{CDCl}_{3}\right) \delta=7.96$ $\left(1 \mathrm{H}, \mathrm{t},{ }^{4} \mathrm{~J} 1.8, \mathrm{C} 6 H\right), 7.68\left(1 \mathrm{H}, \mathrm{t},{ }^{4} \mathrm{~J} 2.2, \mathrm{C} 2 \mathrm{H}\right), 7.37\left(1 \mathrm{H}, \mathrm{dd},{ }^{4} \mathrm{~J}\right.$ 2.1, 1.8, C4H), $3.89\left(3 \mathrm{H}, \mathrm{s}, \mathrm{OCH}_{3}\right) ;{ }^{13} \mathrm{C} \mathrm{NMR}\left(125 \mathrm{MHz}, \mathrm{CDCl}_{3}\right)$
$\delta=160.6(C 3), 149.5(C 5), 123.9(C 2), 123.0(C 1), 107.8(C 4)$, $56.2\left(\mathrm{OCH}_{3}\right)$. Analytical data is in agreement with literature. ${ }^{17 \mathrm{a}}$

To a solution of 1-bromo-3-methoxy-5-nitrobenzene (6) $(50.0 \mathrm{mmol})$ in $\mathrm{MeOH}: \mathrm{H}_{2} \mathrm{O}(1: 1,150 \mathrm{~mL})$ was added carbonyl iron $(250 \mathrm{mmol}, 14.0 \mathrm{~g})$ and ammonium chloride $(21.4 \mathrm{~g}, 400$ mmol) at RT and mixture was stirred at $95{ }^{\circ} \mathrm{C}$ for $2 \mathrm{~h}$. The mixture was cooled to RT, adjusted to $\mathrm{pH} 8$ with aq. sat. $\mathrm{Na}_{2} \mathrm{CO}_{3}$ and filtered over celite ${ }^{\mathbb{R}}$ (particle size 0.02-0.1 mm). The filtrate was concentrated in vacuo, aqueous residue diluted with $\mathrm{H}_{2} \mathrm{O}$ $(200 \mathrm{~mL})$ and extracted with EtOAc $(3 \times 250 \mathrm{~mL})$. The combined organic layer was dried over $\mathrm{Na}_{2} \mathrm{SO}_{4}$, concentrated and dried in vacuo to give 3-bromo-5-methoxyaniline as a dark solid (7.98 g, $79 \%$ over two steps). $\mathrm{R}_{f} 0.47 \quad\left(\mathrm{CH}_{2} \mathrm{Cl}_{2} \quad 100 \%\right)$; ${ }^{1} \mathrm{H}$ NMR $\left(500 \mathrm{MHz}, \mathrm{CDCl}_{3}\right) \delta=6.46\left(1 \mathrm{H}, \mathrm{t},{ }^{4} J 1.9\right), 6.44(1 \mathrm{H}, \mathrm{t}$, $\left.{ }^{4} J 1.8\right) 6.13\left(1 \mathrm{H}, \mathrm{t},{ }^{4} J 2.1\right), 3.74\left(3 \mathrm{H}, \mathrm{s}, \mathrm{OCH}_{3}\right), 3.70(2 \mathrm{H}, \mathrm{br}$, $\left.\mathrm{NH}_{2}\right) ;{ }^{13} \mathrm{C} \mathrm{NMR}\left(125 \mathrm{MHz}, \mathrm{CDCl}_{3}\right) \delta=161.3(\mathrm{C} 5), 148.6(\mathrm{C} 3)$, $123.3(C 1), 110.9(C 2), 107.3(C 6), 99.9(C 4), 55.3\left(\mathrm{OCH}_{3}\right)$; Analytical data is in agreement with literature. ${ }^{16 \mathrm{c}}$

To a mixture of aq. formaldehyde $(37 \mathrm{wt} \%, 4.47 \mathrm{~mL}$, $60.0 \mathrm{mmol})$ in THF $(15 \mathrm{~mL})$ and aq. $\mathrm{H}_{2} \mathrm{SO}_{4}\left(3.0 \mathrm{molL}^{-1}, 4.00\right.$ $\mathrm{mL}$ ) in an open flask was added via dropping funnel a suspension of 3-bromo-5-methoxyaniline $(3.03 \mathrm{~g}, 15.0 \mathrm{mmol})$ and $\mathrm{NaBH}_{4}$ $(1.70 \mathrm{~g}, 45.0 \mathrm{mmol})$ in THF $(15 \mathrm{~mL})$ over $1 \mathrm{~h}$ at $15{ }^{\circ} \mathrm{C} \pm 5{ }^{\circ} \mathrm{C}$ (inside temperature). After 30 min of stirring, $\mathrm{pH} 8$ was adjusted with aq. sat. $\mathrm{Na}_{2} \mathrm{CO}_{3}$ and concentrated in vacuo. The residue was diluted with water $(80 \mathrm{~mL})$ extracted with $\mathrm{CH}_{2} \mathrm{Cl}_{2}(3 \times 100 \mathrm{~mL})$. The combined organic layer was dried over $\mathrm{Na}_{2} \mathrm{SO}_{4}$ and concentrated in vacuo. The residue was suspended with $\mathrm{CH}_{2} \mathrm{Cl}_{2}$, filtered and the filtrate was concentrated in vacuo to give 3bromo-5-methoxy- $N, N$-dimethylaniline (7) as an orange oil (1.81 g, 52\%): $\mathrm{R}_{f} 0.81\left(\mathrm{CH}_{2} \mathrm{Cl}_{2} 100 \%\right) ; v_{\max }$ (neat): $2935 \mathrm{~m}, 2358 \mathrm{w}$, $1604 \mathrm{~s}, 1557 \mathrm{~s}, 1495 \mathrm{~m}, 1431 \mathrm{~m}, 1358 \mathrm{w}, 1319 \mathrm{w}, 1276 \mathrm{w}, 1238 \mathrm{~m}$, $1149 \mathrm{~m}, \quad 1060 \mathrm{~m}, \quad 996 \mathrm{~m}, \quad 875 \mathrm{w}, 812 \mathrm{w}, \quad 788 \mathrm{~m}, \quad 675 \mathrm{w}$; ${ }^{1} \mathrm{H}$ NMR $\left(500 \mathrm{MHz}, \mathrm{CDCl}_{3}\right) \delta=6.47\left(1 \mathrm{H}, \mathrm{dd},{ }^{4} J 2.2,1.7, \mathrm{C} 2 H\right)$; $6.42\left(1 \mathrm{H}, \mathrm{dd},{ }^{4} J 2.0,1.7, \mathrm{C} 6 H\right), 6.13\left(1 \mathrm{H}, \mathrm{t},{ }^{4} \mathrm{~J} 2.2, \mathrm{C} 4 H\right), 3.77$ $\left(3 \mathrm{H}, \mathrm{s}, \mathrm{OCH}_{3}\right), 2.92\left(6 \mathrm{H}, \mathrm{s}, \mathrm{N}\left(\mathrm{CH}_{3}\right)_{2}\right) ;{ }^{13} \mathrm{C} \mathrm{NMR}(125 \mathrm{MHz}$, $\left.\mathrm{CDCl}_{3}\right) \delta=161.1(C 5), 152.3(C 3), 123.5(C 1), 108.6(C 2)$, 104.7 (C6), 97.7 (C4), $55.4\left(\mathrm{OCH}_{3}\right), 40.4\left(\mathrm{~N}\left(\mathrm{CH}_{3}\right)_{2}\right)$; ESI-MS: $\mathrm{m} / \mathrm{z}$ calcd. for $\mathrm{C}_{9} \mathrm{H}_{13} \mathrm{NO}^{+} 230.0175$ found $230.0173\left[\mathrm{M}+\mathrm{H}^{+}\right]$.

\subsubsection{3-methoxy-N-(3-methoxyphenyl)-N- phenylaniline (1a)}

To a degassed mixture of $\operatorname{Pd}_{2}(\mathrm{dba})_{3}(91.6 \mathrm{mg}, 100 \mu \mathrm{mol})$, RuPhos (95\%, $98.2 \mathrm{mg}, 200 \mu \mathrm{mol})$ and sodium $t$ butoxide $(1.15$ $\mathrm{g}, 12.0 \mathrm{mmol})$ in $\mathrm{PhMe}(20 \mathrm{~mL})$ was added 3-iodoanisole (9) $(1.00 \mathrm{~mL}, 8.40 \mathrm{mmol})$ and aniline $(\boldsymbol{8})(0.365 \mathrm{~mL}, 4.00 \mathrm{mmol})$. The mixture was stirred at $100{ }^{\circ} \mathrm{C}$ for $14 \mathrm{~h}$, then cooled to RT, diluted with water and extracted with $\mathrm{CH}_{2} \mathrm{Cl}_{2}(3 \times 250 \mathrm{~mL})$. The combined organic layer was dried over $\mathrm{Na}_{2} \mathrm{SO}_{4}$ and concentrated in vacuo. Column chromatography over silica gel with eluent pentane: $\mathrm{CH}_{2} \mathrm{Cl}_{2} \quad 4: 1$ to $1: 1$ gave 3-methoxy- $N$-(3methoxyphenyl)- $N$-phenylaniline $(\mathbf{1 a})$ as a brownish oil $(1.02 \mathrm{~g}$, $84 \%): \mathrm{R}_{f} 0.53\left(\mathrm{CH}_{2} \mathrm{Cl}_{2} 100 \%\right) ; v_{\max }$ (neat): 3391w, 3001w, $2954 \mathrm{w}, 2834 \mathrm{w}, 2339 \mathrm{w}, 1582 \mathrm{~s}, 1484 \mathrm{~s}, 1314 \mathrm{~m}, 1273 \mathrm{~m}, 1206 \mathrm{~s}$, $1158 \mathrm{~m}, 1140 \mathrm{~m}, 1043 \mathrm{~m}, 982 \mathrm{w}, 849 \mathrm{~m}, 766 \mathrm{~m}, 747 \mathrm{~m}, 690 \mathrm{~s}$; ${ }^{1} \mathrm{H} \mathrm{NMR}\left(500 \mathrm{MHz}, \mathrm{CDCl}_{3}\right) \delta=7.22-7.26(2 \mathrm{H}, \mathrm{m}, \mathrm{C} 3 H), 7.12-$ $7.15(2 \mathrm{H}, \mathrm{m}, \mathrm{C} 5 H), 7.09-7.11\left(2 \mathrm{H}, \mathrm{m}, \mathrm{C} 2{ }^{\prime} H\right), 6.99-7.03(1 \mathrm{H}, \mathrm{m}$, C4'H), $6.66\left(2 \mathrm{H}, \mathrm{ddd},{ }^{3} J\right.$ 8.0, ${ }^{4} J$ 2.1, 0.9, C6H) $6.63-6.64(2 \mathrm{H}$, $\mathrm{m}, \mathrm{C} 2 \mathrm{H}), 6.56\left(2 \mathrm{H}, \mathrm{ddd},{ }^{3} J 8.2,{ }^{4} J 2.5,0.8, \mathrm{C} 4 H\right), 3.71(6 \mathrm{H}, \mathrm{s}$, $\left.\mathrm{OCH}_{3}\right) ;{ }^{13} \mathrm{C} \mathrm{NMR}\left(125 \mathrm{MHz}, \mathrm{CDCl}_{3}\right) \delta=160.4(\mathrm{C} 3), 149.0(\mathrm{Cl})$, 147.6 (C1'), 129.8 (C5), 129.2 (C3'), 124.7 (C4'), 123.0 (C2'), 116.7 (C6), $110.0(C 2), 108.2(C 4), 55.3\left(\mathrm{OCH}_{3}\right)$; ESI-MS: m/z calcd. for $\mathrm{C}_{20} \mathrm{H}_{20} \mathrm{NO}_{2}{ }^{+} 306.1489$ found $306.1486\left[\mathrm{M}+\mathrm{H}^{+}\right]$.

\subsubsection{5-Methoxy-N -(3-methoxyphenyl)- $N^{3}, N^{3}-$} dimethyl-N $N^{1}$-phenylbenzene-1,3-diamine (1b)

To a degassed mixture of tris(dibenzylideneacetone)dipalladium (19.5 mg, $21.3 \mu \mathrm{mol}), \quad 1,1$ '-bis(diphenylphosphino)ferrocene 
(23.6 mg, $42.5 \mu \mathrm{mol}$ ) and sodium $t$ butoxide $(123 \mathrm{mg}, 1.28 \mathrm{mmol})$ was added 3-bromo-5-methoxy- $N, N$-dimethylaniline (7) (196 $\mathrm{mg}, 0.850 \mathrm{mmol})$ in $\mathrm{PhMe}(1.1 \mathrm{~mL})$ and aniline $(8)(77.6 \mu \mathrm{L}$, $0.850 \mathrm{mmol}$ ) at RT and stirred $12 \mathrm{~h}$ at $100{ }^{\circ} \mathrm{C}$. The mixture was diluted with $\mathrm{H}_{2} \mathrm{O}(8.5 \mathrm{~mL})$ and extracted with $\mathrm{CH}_{2} \mathrm{Cl}_{2}(3$ × 25 $\mathrm{mL}$ ). The combined organic layer was dried over $\mathrm{Na}_{2} \mathrm{SO}_{4}$ and concentrated in vacuo. Column chromatography on silica gel with pentane: $\mathrm{CH}_{2} \mathrm{Cl}_{2}$ 2:1 to 1:1 gave 3-bromo-5-methoxy- $N$ phenylaniline as yellow oil (182 mg, 88\%): $\mathrm{R}_{f} 0.25\left(\mathrm{CH}_{2} \mathrm{Cl}_{2}\right.$ $100 \%) ; v_{\max }$ (neat): $3381 \mathrm{w}, 2934 \mathrm{w}, 1583 \mathrm{~s}, 1495 \mathrm{~m}, 1305 \mathrm{w}$, $1243 \mathrm{w}, 1155 \mathrm{~m}, 1065 \mathrm{w}, 891 \mathrm{w}, 754 \mathrm{~m}, 631 \mathrm{~s} ;{ }^{1} \mathrm{H}$ NMR $(500 \mathrm{MHz}$, $\left.\mathrm{CDCl}_{3}\right) \delta=7.24-7.27\left(2 \mathrm{H}, \mathrm{m}, \mathrm{C} 3^{\prime} H\right), 7.08-7.10(2 \mathrm{H}, \mathrm{m}, \mathrm{C} 2 \mathrm{H}$, $\mathrm{C} 6 H), 6.89-6.92\left(1 \mathrm{H}, \mathrm{m}, \mathrm{C} 4{ }^{\prime} H\right), 6.08-6.09(1 \mathrm{H}, \mathrm{m}, \mathrm{C} 6 H), 6.06-$ $6.07(1 \mathrm{H}, \mathrm{m}, \mathrm{C} 2 H), 5.91-5.92(1 \mathrm{H}, \mathrm{m}, \mathrm{C} 4 H), 5.66(1 \mathrm{H}, \mathrm{br}, \mathrm{NH})$, $3.76\left(3 \mathrm{H}, \mathrm{s}, \mathrm{OCH}_{3}\right), 2.91\left(6 \mathrm{H}, \mathrm{s}, \mathrm{N}\left(\mathrm{CH}_{3}\right)_{2}\right) ;{ }^{13} \mathrm{C} \mathrm{NMR}(125 \mathrm{MHz}$, $\left.\mathrm{CDCl}_{3}\right) \delta=161.5(C 5), 152.6(C 3), 144.8(C 1), 143.3(C 1)$, 129.3 (C2'), 120.8 (C4'), 118.1 (C3'), 95.5 (C2), 92.6 (C4), 92.5 (C6), $55.2\left(\mathrm{OCH}_{3}\right), 40.6\left(\mathrm{~N}\left(\mathrm{CH}_{3}\right)_{2}\right)$; ESI-MS: $\mathrm{m} / \mathrm{z}$ calcd. for $\mathrm{C}_{15} \mathrm{H}_{19} \mathrm{~N}_{2} \mathrm{O}^{+} 243.1492$ found $243.1490\left[\mathrm{M}+\mathrm{H}^{+}\right]$.

To a degassed mixture of $\operatorname{Pd}_{2}(\mathrm{dba})_{3}(16.8 \mathrm{mg}, 18.3 \mu \mathrm{mol})$, RuPhos $(95 \%, 17.9 \mathrm{mg} 36.5 \mu \mathrm{mol})$ and sodium $t$ butoxide $(106$ $\mathrm{mg}, 1.10 \mathrm{mmol}$ ) was added 3-bromo-5-methoxy- $N$-phenylaniline (177 mg, $0.730 \mathrm{mmol})$ in PhMe $(1.4 \mathrm{~mL})$ and 3-iodoanisole (9) $(87.2 \mu \mathrm{L}, 0.730 \mathrm{mmol})$. The mixture was stirred at $100{ }^{\circ} \mathrm{C}$ for $14 \mathrm{~h}$, then cooled to RT, diluted with water and extracted with $\mathrm{CH}_{2} \mathrm{Cl}_{2}(3 \times 10 \mathrm{~mL})$. The combined organic layer was dried over $\mathrm{Na}_{2} \mathrm{SO}_{4}$ and concentrated in vacuo. Column chromatography over silica gel with eluent pentane: $\mathrm{CH}_{2} \mathrm{Cl}_{2} 2: 1$ to $1: 1$ to $1: 2$ gave a brownish oil 5-methoxy- $N^{1}$-(3-methoxyphenyl)- $N^{3}, N^{3}$-dimethyl$N^{1}$-phenylbenzene-1,3-diamine (1b) (250 mg, 98\%): $\mathrm{R}_{f} 0.58$ $\left(\mathrm{CH}_{2} \mathrm{Cl}_{2} 100 \%\right) ; v_{\max }$ (neat): $2934 \mathrm{w}, 1588 \mathrm{~s}, 1489 \mathrm{~m}, 1315 \mathrm{w}$, $1304 \mathrm{w}, 1270 \mathrm{~m}, 1244 \mathrm{~m}, 1206 \mathrm{~m}, 1165 \mathrm{~m}, 1146 \mathrm{~m}, 1064 \mathrm{w}, 813 \mathrm{w}$, $697 \mathrm{~m} ;{ }^{1} \mathrm{H}$ NMR $\left(500 \mathrm{MHz}, \mathrm{CDCl}_{3}\right) \delta=7.21-7.24(2 \mathrm{H}, \mathrm{m}, \mathrm{C} 2 " \mathrm{H}$, C4"H), 7.10-7.13 (3H, m, C5' H, C1" $H$, C6" $H), 6.96-6.99(1 \mathrm{H}$, m, C3” H), 6.67-6.69 (1H, m, C6'H), 6.65-6.66 (1H, m, C2'H), 6.52-6.54 (1H, m, C4'H), 6.10-6.11 (1H, m, C2H), 6.03-6.04 $(1 \mathrm{H}, \mathrm{m}, \mathrm{C} 6 H), 5.99-6.00(1 \mathrm{H}, \mathrm{m}, \mathrm{C} 4 H), 3.71\left(3 \mathrm{H}, \mathrm{s}, \mathrm{C} 3{ }^{\prime} \mathrm{OCH}_{3}\right)$, $3.69\left(3 \mathrm{H}, \mathrm{s}, \mathrm{C} 5 \mathrm{OCH}_{3}\right), 2.84\left(6 \mathrm{H}, \mathrm{s}, \mathrm{N}\left(\mathrm{CH}_{3}\right)_{2}\right) ;{ }^{13} \mathrm{C} \mathrm{NMR}(125$ $\left.\mathrm{MHz}, \mathrm{CDCl}_{3}\right) \delta=161.2\left(\mathrm{C}^{\prime}\right.$ '), 160.3 (C5), 152.2 (C3), 149.3 (C1), 149.2 (C1'), 147.8 (C1'), 129.5 (C5'), 129.0 (C3”, C5”) 124.3 (C2", C6"), 122.5 (C4”), 116.4 (C6'), 109.5 (C2'), 107.7 (C4'), 102.9 (C2), 99.3 (C6), 94.5 (C4), $55.2\left(\mathrm{OCH}_{3}\right), 55.2$ $\left(\mathrm{OCH}_{3}\right), 40.6\left(\mathrm{~N}\left(\mathrm{CH}_{3}\right)_{2}\right)$; ESI-MS: $\mathrm{m} / \mathrm{z}$ calcd. for $\mathrm{C}_{22} \mathrm{H}_{25} \mathrm{~N}_{2} \mathrm{O}_{2}{ }^{+}$ 349.1911 found $349.1909\left[\mathrm{M}+\mathrm{H}^{+}\right]$.

4.1.4. $N^{1}-(3-($ dimethylamino)-5-methoxyphenyl)-5methoxy- $N^{3}, N^{3}$-dimethyl-N $N^{1}$ - phenylbenzene-1,3diamine (1c)

To a degassed mixture of 3-bromo-5-methoxy- $N, N$ dimethylaniline (7) (920 mg, $4.00 \mathrm{mmol})$, RuPhos (95\%, $98.2 \mathrm{mg}, 200 \mu \mathrm{mol}), \mathrm{Pd}_{2}(\mathrm{dba})_{3}(91.6 \mathrm{mg}, 100 \mu \mathrm{mol})$ and sodium $t$ butoxide $(577 \mathrm{mg}, 6.00 \mathrm{mmol})$ in PhMe $(10 \mathrm{~mL})$ at RT was added aniline $(\boldsymbol{8})(183 \mu \mathrm{L}, 2.00 \mathrm{mmol})$. The mixture was stirred at $100{ }^{\circ} \mathrm{C}$ for $12 \mathrm{~h}$, then cooled to $\mathrm{RT}$, diluted with water and extracted with $\mathrm{CH}_{2} \mathrm{Cl}_{2}(3 \times 150 \mathrm{~mL})$. The combined organic layer was dried over $\mathrm{Na}_{2} \mathrm{SO}_{4}$ and concentrated in vacuo. Column chromatography over silica gel with eluent pentane: $\mathrm{CH}_{2} \mathrm{Cl}_{2} 3: 1$ to $1: 1$ to $100 \% \quad \mathrm{CH}_{2} \mathrm{Cl}_{2}$ gave $N^{l}$-(3-(dimethylamino)-5methoxyphenyl)-5-methoxy- $N^{3}, N^{3}$-dimethyl- $N^{I}$-phenylbenzene1,3-diamine (1c) (365 mg, $47 \%,{ }^{18}$ m.p. $\left.118.8-120.6{ }^{\circ} \mathrm{C}\right)$ as a beige solid: $\mathrm{R}_{f} 0.53$ (pentane: $\mathrm{CH}_{2} \mathrm{Cl}_{2} 1: 1$ ); $v_{\max }$ (neat): $2934 \mathrm{w}$, $2339 \mathrm{w}, 1578 \mathrm{~s}, 1491 \mathrm{~m}, 1447 \mathrm{~m}, 1297 \mathrm{w}, 1269 \mathrm{~m}, 1242 \mathrm{w}, 1202 \mathrm{w}$, $1173 \mathrm{w}, 1146 \mathrm{~m}, 1066 \mathrm{~m}, 765 \mathrm{~m}, 712 \mathrm{~m}, 630 \mathrm{~m} ;{ }^{1} \mathrm{H}$ NMR $(500 \mathrm{MHz}$, $\left.\mathrm{CDCl}_{3}\right) \delta=7.18-7.22\left(2 \mathrm{H}, \mathrm{m}, \mathrm{C} 3^{\prime} H\right), 7.11-7.13\left(2 \mathrm{H}, \mathrm{m}, \mathrm{C} 2{ }^{\prime} H\right)$, 6.93-6.97 (1H, m, C4'H), $6.14\left(2 \mathrm{H}, \mathrm{t},{ }^{4} J 2.0, \mathrm{C} 2 H\right), 6.06(2 \mathrm{H}, \mathrm{t}$, $\left.{ }^{4} J 2.0, \mathrm{C} 6 H\right), 5.97\left(2 \mathrm{H}, \mathrm{t},{ }^{4} J 1.9, \mathrm{C} 4 H\right), 3.69\left(6 \mathrm{H}, \mathrm{s}, 2 \times \mathrm{OCH}_{3}\right)$, $2.84\left(12 \mathrm{H}, \mathrm{s}, 2 \times \mathrm{N}\left(\mathrm{CH}_{3}\right)_{2}\right) ;{ }^{13} \mathrm{C} \mathrm{NMR}\left(125 \mathrm{MHz}, \mathrm{CDCl}_{3}\right) \delta=$ 161.1 (C5), 152.1 (C3), 149.4 (C1), 148.0 (C1'), 128.8 (C3'),
124.2 (C2'), 122.1 (C4'), 102.6 (C2), 98.9 (C6), 94.2 (C4), 55.2 $\left(\mathrm{OCH}_{3}\right), 40.7\left(2 \mathrm{x} \mathrm{N}\left(\mathrm{CH}_{3}\right)_{2}\right)$; ESI-MS: $\mathrm{m} / \mathrm{z}$ calcd. for $\mathrm{C}_{24} \mathrm{H}_{30} \mathrm{~N}_{3} \mathrm{O}_{2}{ }^{+} 392.2333$ found $392.2330\left[\mathrm{M}+\mathrm{H}^{+}\right]$.

\subsubsection{3-Methoxy-N-(3-methoxyphenyl)-N- methylaniline $(\mathbf{1} \boldsymbol{d})$}

Prepared according to modified literature procedures: ${ }^{19}$ To a degassed mixture of tris(dibenzylideneacetone)dipalladium( $(0)$ (458 mg, $0.500 \mathrm{mmol}), 1,1$ '-bis(diphenyl-phosphino)ferrocene (554 $\mathrm{mg}, 1.00 \mathrm{mmol})$ and sodium $t$ butoxide $(2.88 \mathrm{~g}, 30.0 \mathrm{mmol})$ in PhMe $(25 \mathrm{~mL})$ was added 3-iodoanisole (9) $(2.38 \mathrm{~mL}, 20.0$ $\mathrm{mmol})$ and $m$-anisidine (10) $(2.24 \mathrm{~mL}, 20.0 \mathrm{mmol})$ at RT and stirred $14 \mathrm{~h}$ at $110{ }^{\circ} \mathrm{C}$. The mixture was diluted with $\mathrm{H}_{2} \mathrm{O}(200$ $\mathrm{mL})$ and extracted with $\mathrm{CH}_{2} \mathrm{Cl}_{2}(3 \times 250 \mathrm{~mL})$. The combined organic layer was dried over $\mathrm{Na}_{2} \mathrm{SO}_{4}$ and concentrated in vacuo. Column chromatography on silica gel with pentane: $\mathrm{CH}_{2} \mathrm{Cl}_{2}$ 1:1 to 1:2 gave bis(3-methoxyphenyl)amine as yellow oil (3.60 g, $79 \%): \mathrm{R}_{f} 0.53\left(\mathrm{CH}_{2} \mathrm{Cl}_{2} 100 \%\right) ; v_{\max }$ (neat): $3389 \mathrm{w}, 2955 \mathrm{w}$, $2836 \mathrm{w}, 2359 \mathrm{w}, 1593 \mathrm{~s}, 1492 \mathrm{~m}, 1273 \mathrm{w}, 1209 \mathrm{~m}, 1158 \mathrm{~m}, 1045 \mathrm{w}$, $968 \mathrm{w}, 835 \mathrm{w}, 765 \mathrm{w}, 687 \mathrm{w} ;{ }^{1} \mathrm{H}$ NMR $\left(500 \mathrm{MHz}, \mathrm{CDCl}_{3}\right) \delta=7.17$ $\left(2 \mathrm{H}, \mathrm{t},{ }^{3} J 8.1, \mathrm{C} 5 H\right), 6.67$ (2H, ddd, $\left.{ }^{3} J 8.1,{ }^{4} J 2.2,0.9, \mathrm{C} 6 H\right)$, 6.65-6.66 (2H, m, C2H), 6.49 (2H, ddd, $\left.{ }^{3} J 8.2,{ }^{4} J 2.4,0.9, \mathrm{C} 4 H\right)$, $5.71(1 \mathrm{H}$, br, $\mathrm{NH}), 3.78\left(6 \mathrm{H}, \mathrm{s}, \mathrm{OCH}_{3}\right) ;{ }^{13} \mathrm{C} \mathrm{NMR}(125 \mathrm{MHz}$, $\left.\mathrm{CDCl}_{3}\right) \delta=160.7(C 3), 144.3(C 1), 130.1$ (C5), $110.6(C 6)$, $106.5(C 4), 103.8(C 2), 55.2\left(\mathrm{OCH}_{3}\right)$; ESI-MS: $\mathrm{m} / \mathrm{z}$ calcd. for $\mathrm{C}_{14} \mathrm{H}_{16} \mathrm{NO}_{2}{ }^{+} 230.1176$ found $230.1175\left[\mathrm{M}+\mathrm{H}^{+}\right]$.

To a solution of bis(3-methoxyphenyl)amine (1.15 g, $5.00 \mathrm{mmol})$ in THF $(20 \mathrm{~mL})$ at RT was added sodium hydride (60\% dispersion in mineral oil, $550 \mathrm{mg}, 13.8 \mathrm{mmol})$. The suspension was heated to $75{ }^{\circ} \mathrm{C}$ and stirred for $30 \mathrm{~min}$ at this temperature. Iodomethane $(0.716 \mathrm{~mL}, 11.5 \mathrm{mmol})$ was added within $5 \mathrm{~min}$ at $75{ }^{\circ} \mathrm{C}$ and the reaction mixture was continued to stir for $2 \mathrm{~h}$ at this temperature. The suspension was treated with water $(20 \mathrm{~mL})$ and extracted with $\mathrm{Et}_{2} \mathrm{O}(3 \times 65 \mathrm{~mL})$. The combined organic layer was dried over $\mathrm{Na}_{2} \mathrm{SO}_{4}$ and concentrated in vacuo. Column chromatography on silica gel with pentane: $\mathrm{CH}_{2} \mathrm{Cl}_{2} \quad 3: 1$ to $1: 1$ gave 3-methoxy- $N$-(3methoxyphenyl)- $N$-methylaniline (1d) as a yellowish oil (979 $\mathrm{mg}, 81 \%): \mathrm{R}_{f} 0.64\left(\mathrm{CH}_{2} \mathrm{Cl}_{2} 100 \%\right) ; v_{\max }$ (neat): $2938 \mathrm{w}, 2834 \mathrm{w}$, $2338 \mathrm{w}, 1589 \mathrm{~s}, 1489 \mathrm{~s}, 1347 \mathrm{w}, 1279 \mathrm{w}, 1221 \mathrm{~m}, 1169 \mathrm{w}, 1122 \mathrm{w}$, $1046 \mathrm{w}, 766 \mathrm{~s}, 708 \mathrm{w}, 631 \mathrm{~s} ;{ }^{1} \mathrm{H}$ NMR $\left(500 \mathrm{MHz}, \mathrm{CDCl}_{3}\right) \delta=7.15$ $7.19(2 \mathrm{H}, \mathrm{m}, \mathrm{C} 5 H), 6.62\left(2 \mathrm{H}\right.$, ddd, $\left.{ }^{3} J 8.1,{ }^{4} J 2.2,0.8, \mathrm{C} 6 H\right), 6.57-$ $6.58(2 \mathrm{H}, \mathrm{m}, \mathrm{C} 2 \mathrm{H}), 6.52\left(2 \mathrm{H}, \mathrm{ddd},{ }^{3} J 8.2,{ }^{4} \mathrm{~J} 2.5,0.8, \mathrm{C} 4 H\right), 3.76$ $\left(6 \mathrm{H}, \mathrm{s}, \mathrm{OCH}_{3}\right), 3.29\left(3 \mathrm{H}, \mathrm{s}, \mathrm{NCH}_{3}\right) ;{ }^{13} \mathrm{C} \mathrm{NMR}\left(125 \mathrm{MHz}, \mathrm{CDCl}_{3}\right)$ $\delta=160.5(C 3), 150.2(C 1), 129.8$ (C5), 113.3 (C6), 106.7 (C4), $106.6(C 2), 55.2\left(\mathrm{OCH}_{3}\right), 40.3\left(\mathrm{NCH}_{3}\right)$; ESI-MS: m/z calcd. for $\mathrm{C}_{15} \mathrm{H}_{18} \mathrm{NO}_{2}{ }^{+} 244.1332$ found $244.1330\left[\mathrm{M}+\mathrm{H}^{+}\right]$.

\subsection{General Procedure A: Double directed ortho-Metalation}

To a solution of bis(3-methoxyphenyl)-amine $\mathbf{l a}$-d $(160 \mu \mathrm{mol})$ in $n$ hexane $(2.0 \mathrm{~mL})$ was added a solution of $n$ butyllithium in hexanes $\left(176 \mu \mathrm{L}, 1.49 \mathrm{molL}^{-1}, 320 \mu \mathrm{mol}\right)$ at RT. The mixture was stirred $6 \mathrm{~h}$ at $65^{\circ} \mathrm{C}$. The reaction mixture was directly used in the next step.

\subsection{General Procedure B: Ester to Acridinium Transformation}

To the reaction mixture of the metalated aryl aniline $\mathbf{1} \boldsymbol{a}^{\prime} \boldsymbol{-} \boldsymbol{d}$ ' in $n$ hexane $(160 \mu \mathrm{mol})$ at $-20^{\circ} \mathrm{C}$ was added a solution of carboxylic acid ester (1) $(100 \mu \mathrm{mol})$ in anhydrous THF $(0.60$ $\mathrm{mL}$ ) and the reaction mixture was allowed to warm to RT over 12 $\mathrm{h}$ or $14 \mathrm{~h}$ (indicated individually). Aqueous $\mathrm{HBr}(1.00 \mathrm{~mL}, 48 \%)$ was added, followed by water $(20 \mathrm{~mL})$ and the mixture was 
extracted by $\mathrm{CHCl}_{3}: i \mathrm{PrOH}$ solution $(4 \mathrm{x} 10 \mathrm{~mL} ; 85: 15)$. The combined organic layer was dried over $\mathrm{Na}_{2} \mathrm{SO}_{4}$, filtered and concentrated in vacuo. Column chromatography with $100 \%$ $\mathrm{CH}_{2} \mathrm{Cl}_{2}$ to $\mathrm{CH}_{2} \mathrm{Cl}_{2}: \mathrm{MeOH} 100: 2$ to $100: 5$ to $100: 8$ to 100:9 gave the product.

\subsection{Synthesis of Acridinium Dyes and 9,10-Dihydroacridin-9-ols}

\subsubsection{1,8-Dimethoxy-9,10-diphenylacridinium} bromide salt $\left(4 \boldsymbol{a}-\boldsymbol{B r}^{-}\right)$

The compound was prepared according to the general procedure A and B using 3-methoxy- $N$-(3-methoxyphenyl)- $N$ phenylaniline (1a) $(48.9 \mathrm{mg}, 160 \mu \mathrm{mol})$ and methyl benzoate $(13.6 \mathrm{mg}, 100 \mu \mathrm{mol})$ and was stirred $12 \mathrm{~h}$ at RT. Purification gave a brown red solid $(20.1 \mathrm{mg}, 43 \%$, HPLC purity: 95.2\%; ${ }^{19}$ decomp. at $\left.115{ }^{\circ} \mathrm{C}\right): \mathrm{R}_{f} 0.19\left(\mathrm{CH}_{2} \mathrm{Cl}_{2}: \mathrm{MeOH} 10: 1\right)$; $v_{\max }$ (neat): $2999 \mathrm{w}, 1586 \mathrm{~s}, 1462 \mathrm{~s}, 1363 \mathrm{~m}, 1265 \mathrm{~s}, 1248 \mathrm{~s}, 1198 \mathrm{w}$, $1082 \mathrm{~s}, 982 \mathrm{w}, 925 \mathrm{w}, 811 \mathrm{~m}, 758 \mathrm{~s}, 738 \mathrm{~s}, 696 \mathrm{~s}, 655 \mathrm{~m} ;{ }^{1} \mathrm{H}$ NMR $\left(500 \mathrm{MHz}, \mathrm{CDCl}_{3}\right): \delta=8.02\left(2 \mathrm{H}, \mathrm{t},{ }^{3} \mathrm{~J} 8.4, \mathrm{C} 3 H, \mathrm{C} 6 H\right), 7.89$ 7.91 (2H, m, C3" H, C5"H), 7.83-7.86 (1H, m, C4"H), 7.64-7.66 $\left(2 \mathrm{H}, \mathrm{m}, \mathrm{C} 2{ }^{\prime} H, \mathrm{C} 6{ }^{\prime} H\right), 7.44-7.50\left(3 \mathrm{H}, \mathrm{m}, \mathrm{C} 3 ' H, \mathrm{C} 4{ }^{\prime} H, \mathrm{C} 5 ' H\right)$, 7.33-7.34 (2H, m, C2'H, C6'H), 7.03 (2H, d, $\left.{ }^{3} J 7.9, \mathrm{C} 2 H, \mathrm{C} 7 H\right)$, $6.92\left(2 \mathrm{H}, \mathrm{dd},{ }^{3} \mathrm{~J} 8.9,{ }^{4} \mathrm{~J} 0.6, \mathrm{C} 4 \mathrm{H}, \mathrm{C} 5 \mathrm{H}\right), 3.52(6 \mathrm{H}, \mathrm{s}, 2 \mathrm{x} \mathrm{OCH})$; ${ }^{13} \mathrm{C}$ NMR $\left(125 \mathrm{MHz}, \mathrm{CDCl}_{3}\right): \delta=163.3(\mathrm{C} 9), 160.2(\mathrm{Cl}, \mathrm{C} 8)$, $142.8(C 4 a, C 10 a), 141.3$ (C1'), 140.4 (C3,C6), 138.4 (C1'), 131.8 (C3”, C5”), 131.6 (C4”), 127.9 (C2”, C6”), 127.2 (C4'), 126.9 (C3', $C 5^{\prime}$ ), 125.7 (C2', C6'), 119.1 (C8a, C9a), 110.9 (C4, $C 5), 107.1(C 2, C 7), 56.9\left(2 \times \mathrm{OCH}_{3}\right)$; ESI-MS: $\mathrm{m} / \mathrm{z}$ calcd. for $\mathrm{C}_{27} \mathrm{H}_{22} \mathrm{NO}_{2}{ }^{+} 392.1645$ found $392.1648\left[\mathrm{M}^{+}\right]$. Luminescence spectroscopy (in MeCN): $\lambda_{\text {abs1 }}: 503 \mathrm{~nm} ; \lambda_{\text {abs } 2}: 409 \mathrm{~nm} ; \varepsilon_{\text {abs1 }}$ : $4.4 \cdot 10^{3} \mathrm{~L} \cdot \mathrm{cm} \cdot \mathrm{mol}^{-1} ; \varepsilon_{\text {abs } 2}: 5.8 \cdot 10^{3} \mathrm{~L} \cdot \mathrm{cm} \cdot \mathrm{mol}^{-1}, \lambda_{\text {em }}(\operatorname{exc} 493): 595$ $\mathrm{nm} ; \lambda_{\text {em }}$ (exc 399): $591 \mathrm{~nm}$; Stokes shift: $92 \mathrm{~nm}$; $\mathrm{E}_{0,0}: 2.23 \mathrm{eV}$; $<\tau_{\mathrm{F}}>$ : $3.1 \mathrm{~ns}$; Cyclic voltammetry (vs SCE): $\mathrm{E}_{1 / 2}\left(\mathrm{P}^{*} / \mathrm{P}^{-}\right):+1.76 \mathrm{~V}$, $\mathrm{E}_{1 / 2}\left(\mathrm{P} / \mathrm{P}^{-}\right):-0.47 \mathrm{~V}$.

\subsubsection{1,8-Dimethoxy-9,10-diphenylacridinium tetrafluoroborate salt $\left(\mathbf{4} \boldsymbol{a}-\boldsymbol{B} \boldsymbol{F}_{4}{ }^{-}\right)$}

The compound was prepared according to the general procedure A and B using 3-methoxy- $N$-(3-methoxyphenyl)- $N$ phenylaniline (1a) (48.9 $\mathrm{mg}, 160 \mu \mathrm{mol})$ and methyl benzoate $(13.6 \mathrm{mg}, 100 \mu \mathrm{mol})$ and was stirred $12 \mathrm{~h}$ at RT and treated with aq. $\mathrm{HBF}_{4}(50 \%, 1.00 \mathrm{~mL})$ instead of aq. $\mathrm{HBr}$. Purification gave a brown red solid (21.3 mg, 44\%, HPLC purity: 97.2\%; decomp. at $\left.140{ }^{\circ} \mathrm{C}\right): \mathrm{R}_{f} 0.28\left(\mathrm{CH}_{2} \mathrm{Cl}_{2}: \mathrm{MeOH} 10: 1\right)$; $v_{\max }$ (neat): $3060 \mathrm{w}, 2938 \mathrm{w}, 1581 \mathrm{~m}, 1501 \mathrm{~m}, 1464 \mathrm{~m}, 1434 \mathrm{~m}$, $1362 \mathrm{~m}, 1266 \mathrm{~s}, 1198 \mathrm{w}, 1048 \mathrm{~s}, 910 \mathrm{w}, 819 \mathrm{w}, 748 \mathrm{~s}, 698 \mathrm{~m} ;{ }^{1} \mathrm{H}$ NMR $\left(600 \mathrm{MHz}, \mathrm{CDCl}_{3}\right): \delta=7.94\left(2 \mathrm{H}, \mathrm{dd},{ }^{3} J 8.9,8.1, \mathrm{C} 3 \mathrm{H}\right.$, $\mathrm{C} 6 H), 7.82-7.88(3 \mathrm{H}, \mathrm{m}, \mathrm{C} 3$ " $H, \mathrm{C} 4$ " $H, \mathrm{C} 5 " H), 7.55-7.56(2 \mathrm{H}$, m, C2" $H, \mathrm{C} 6 " H), 7.42-7.48\left(3 \mathrm{H}, \mathrm{m}, \mathrm{C} 3{ }^{\prime} H, \mathrm{C} 4{ }^{\prime} H, \mathrm{C} 5 ' H\right), 7.31-$ $7.33\left(2 \mathrm{H}, \mathrm{m}, \mathrm{C} 2^{\prime} H, \mathrm{C} 6{ }^{\prime} H\right), 6.98\left(2 \mathrm{H}, \mathrm{d},{ }^{3} J 8.0, \mathrm{C} 2 H, \mathrm{C} 7 H\right), 6.89$ $\left(2 \mathrm{H}, \mathrm{d},{ }^{3} \mathrm{~J} 9.0, \mathrm{C} 4 \mathrm{H}, \mathrm{C} 5 \mathrm{H}\right), 3.49\left(6 \mathrm{H}, \mathrm{s}, 2 \times \mathrm{OCH}_{3}\right) ;{ }^{13} \mathrm{C} \mathrm{NMR}$ $\left(151 \mathrm{MHz}, \mathrm{CDCl}_{3}\right): \delta=163.4(C 9), 160.2(C 1, C 8), 142.7(C 4 a$, C10a), 141.4 (C1'), 140.9 (C3, C6), 138.4 (C1'), 131.7 (C3”, C5”), 131.5 (C4'), 127.8 (C2", C6”), 127.1 (C4'), 126.9 (C3', $\left.C 5^{\prime}\right), 125.6$ (C2', C6'), 119.1 (C8a, C9a), 110.8 (C4, C5), 106.9 $(C 2, C 7), 56.7\left(2 \times \mathrm{OCH}_{3}\right) ;{ }^{19} \mathrm{~F} \mathrm{NMR}\left(235 \mathrm{MHz}, \mathrm{CDCl}_{3}\right):-154.5$; ESI-MS: $\mathrm{m} / \mathrm{z}$ calcd. for $\mathrm{C}_{27} \mathrm{H}_{22} \mathrm{NO}_{2}{ }^{+} 392.1645$ found 392.1649 $\left[\mathrm{M}^{+}\right]$. Luminescence spectroscopy (in $\mathrm{MeCN}$ ): $\lambda_{\text {absl }}: 503 \mathrm{~nm}$; $\lambda_{\text {abs2 }}: 409 \mathrm{~nm} ; \varepsilon_{\text {abs } 1}: 3.2 \cdot 10^{3} \mathrm{~L} \cdot \mathrm{cm} \cdot \mathrm{mol}^{-1} ; \varepsilon_{\text {abs } 2}: 4.2 \cdot 10^{3} \mathrm{~L} \cdot \mathrm{cm} \cdot \mathrm{mol}^{-}$ $1, \lambda_{\text {em }}$ (exc 493): $596 \mathrm{~nm} ; \lambda_{\text {em }}$ (exc 399): $592 \mathrm{~nm}$; Stokes shift: 93 $\mathrm{nm} ; \mathrm{E}_{0,0}: 2.23 \mathrm{eV} ;\left\langle\tau_{\mathrm{F}}>: 3.4 \mathrm{~ns} ;\right.$ Cyclic voltammetry (vs SCE): $\mathrm{E}_{1 / 2}\left(\mathrm{P}^{*} / \mathrm{P}^{-}\right):+1.74 \mathrm{~V}, \mathrm{E}_{1 / 2}\left(\mathrm{P} / \mathrm{P}^{-}\right):-0.49 \mathrm{~V}$.

\subsubsection{3-(Dimethylamino)-1,8-dimethoxy-9,10-} diphenylacridinium bromide salt (4b)

The compound was prepared according to the general procedure $\mathrm{A}$ and $\mathrm{B}$ using 5-methoxy- $N^{1}$-(3-methoxyphenyl)- $N^{3}, N^{3}$ dimethyl- $N^{1}$-phenylbenzene-1,3-diamine (1b) (55.8 mg, $160 \mu \mathrm{mol})$ and methyl benzoate $(13.6 \mathrm{mg}, 100 \mu \mathrm{mol})$ and was stirred $14 \mathrm{~h}$ at RT. Purification gave a brown red solid (13.6 mg, 26\%, HPLC purity: $94.7 \% ;^{19}$ decomp. at $\left.117^{\circ} \mathrm{C}\right): \mathrm{R}_{f} 0.18$ $\left(\mathrm{CH}_{2} \mathrm{Cl}_{2}: \mathrm{MeOH} 10: 1\right)$; $v_{\max }$ (neat): 3387w, 2926m, 2361m, $2178 \mathrm{w}, 1623 \mathrm{~s}, 1597 \mathrm{~s}, 1501 \mathrm{~s}, 1428 \mathrm{~s}, 1373 \mathrm{~m}, 1349 \mathrm{~m}, 1295 \mathrm{~m}$, $1255 \mathrm{~s}, 1182 \mathrm{~m}, 1096 \mathrm{~s}, 973 \mathrm{~m}, 921 \mathrm{~m}, 806 \mathrm{w}, 771 \mathrm{~m}, 723 \mathrm{~s}, 697 \mathrm{~s}$, $652 \mathrm{w} ;{ }^{1} \mathrm{H}$ NMR $\left(500 \mathrm{MHz}, \mathrm{CDCl}_{3}\right): \delta=7.84-7.87(2 \mathrm{H}, \mathrm{m}$, C3"'H, C5"H), 7.76-7.79 (1H, m, C4'H), 7.58 (1H, dd, ${ }^{3} J$ 8.6, 8.3, C6H), 7.38-7.43 (5H, m, C3'H, C4'H, C5'H, C2'”H, C6”H), $7.20\left(2 \mathrm{H}, \mathrm{dd},{ }^{3} J 7.5,{ }^{4} J 1.3, \mathrm{C} 2{ }^{\prime} \mathrm{H}, \mathrm{C} 6{ }^{\prime} \mathrm{H}\right), 6.71\left(1 \mathrm{H}, \mathrm{d},{ }^{3} J 8.0\right.$, $\mathrm{C} 7 \mathrm{H}), 6.51\left(1 \mathrm{H}, \mathrm{d},{ }^{3} J 8.6, \mathrm{C} 5 \mathrm{H}\right), 6.41\left(1 \mathrm{H}, \mathrm{d},{ }^{4} J 1.1, \mathrm{C} 2 \mathrm{H}\right), 5.42$ $\left(1 \mathrm{H}, \mathrm{d},{ }^{4} \mathrm{~J} 1.1, \mathrm{C} 4 \mathrm{H}\right), 3.52\left(3 \mathrm{H}, \mathrm{s}, \mathrm{OCH}_{3}\right), 3.38\left(3 \mathrm{H}, \mathrm{s}, \mathrm{OCH}_{3}\right)$, $3.19\left(6 \mathrm{H}\right.$, br, $\left.\mathrm{N}\left(\mathrm{CH}_{3}\right)_{2}\right) ;{ }^{13} \mathrm{C} \mathrm{NMR}\left(125 \mathrm{MHz}, \mathrm{CDCl}_{3}\right): \delta=161.3$ (C1), 160.0 (C8), 157.3 (C3), 156.1 (C9), 145.4 (C4a), 142.0 (C10a), 141.6 (C1'), 138.7 (C1'), 136.2 (C6), 132.0 (C3', C5'), 131.0 (C4”), 128.0 (C2', C6”), 126.8 (C3', C5'), 126.7 (C4'), 125.9 (C2', C6'), 115.5 (C9a), 114.7 (C8a), 109.6 (C5), 105.7 (C7), 95.8 (C2), $89.1(C 4), 57.0\left(\mathrm{C} 1 O C H_{3}\right), 56.3\left(\mathrm{C}^{2} \mathrm{OCH}_{3}\right), 41.3$ $\left(\mathrm{N}\left(\mathrm{CH}_{3}\right)_{2}\right)$; ESI-MS: $\mathrm{m} / \mathrm{z}$ calcd. for $\mathrm{C}_{29} \mathrm{H}_{27} \mathrm{~N}_{2} \mathrm{O}_{2}{ }^{+} 435.2067$ found $435.2073\left[\mathrm{M}^{+}\right]$. Luminescence spectroscopy (in MeCN): $\boldsymbol{\lambda}_{\text {absl }}$ : $501 \mathrm{~nm} ; \boldsymbol{\lambda}_{\mathrm{abs} 2}: 430 \mathrm{~nm} ; \boldsymbol{\varepsilon}_{\mathrm{abs} 1}: 8.6 \cdot 10^{3} \mathrm{~L} \cdot \mathrm{cm} \cdot \mathrm{mol}^{-1} ; \boldsymbol{\varepsilon}_{\mathrm{abs} 2}$ : $1.6 \cdot 10^{4} \mathrm{~L} \cdot \mathrm{cm} \cdot \mathrm{mol}^{-1}, \lambda_{\text {em }}($ exc 491$): 584 \mathrm{~nm} ; \lambda_{\text {em }}($ exc 420$): 589$ $\mathrm{nm}$; Stokes shift: $83 \mathrm{~nm} ; \mathrm{E}_{0,0}: 2.25 \mathrm{eV} ;\left\langle\tau_{\mathrm{F}}\right\rangle: 4.7 \mathrm{~ns}$; Cyclic voltammetry (vs SCE): $\mathrm{E}_{1 / 2}\left(\mathrm{P}^{*} / \mathrm{P}^{-}\right):+1.31 \mathrm{~V}, \mathrm{E}_{1 / 2}\left(\mathrm{P} / \mathrm{P}^{-}\right):-0.94 \mathrm{~V}$.

\subsubsection{3,6-Bis (dimethylamino)-1,8-dimethoxy-9,10- diphenylacridinium bromide salt (4c)}

The compound was prepared according to the general procedure $\mathrm{A}$ and $\mathrm{B}$ using $N^{l}$-(3-(dimethylamino)-5methoxyphenyl)-5-methoxy- $N^{3}, N^{3}$-dimethyl- $N^{I}$-phenylbenzene1,3-diamine (1c) $(62.6 \mathrm{mg}, 160 \mu \mathrm{mol})$ in $n$ hexane: $\mathrm{Et}_{2} \mathrm{O}(2.2 \mathrm{~mL}$, $10: 1)$ and methyl benzoate $(13.6 \mathrm{mg}, 100 \mu \mathrm{mol})$ and was stirred $14 \mathrm{~h}$ at RT. Purification gave a brown red solid (19.7 mg, 35\%, HPLC purity: $78.7 \%$; 19 decomp. at $\left.148{ }^{\circ} \mathrm{C}\right): \mathrm{R}_{f} \quad 0.17$ $\left(\mathrm{CH}_{2} \mathrm{Cl}_{2}: \mathrm{MeOH} 10: 1\right) ; v_{\max }$ (neat): 2925w, 2360w, 2166w, 1599s, $1490 \mathrm{~m}, 1433 \mathrm{w}, 1333 \mathrm{~m}, 1254 \mathrm{~s}, 975 \mathrm{w}, 923 \mathrm{w}, 781 \mathrm{~m}, 630 \mathrm{w} ;{ }^{1} \mathrm{H}$ NMR $\left(500 \mathrm{MHz}, \mathrm{CDCl}_{3}\right): \delta=7.82-7.85(2 \mathrm{H}, \mathrm{m}), 7.73-7.76(1 \mathrm{H}$, m), 7.38-7.40 (4H, m, C2' $H, \mathrm{C} 6$ ' $H, \mathrm{C} 2$ " $H, \mathrm{C} 6 " H), 7.33-7.36$ $(1 \mathrm{H}, \mathrm{m}), 7.17-7.18(2 \mathrm{H}, \mathrm{m}), 6.07\left(2 \mathrm{H}, \mathrm{d},{ }^{4} \mathrm{~J} 1.9, \mathrm{C} 2 \mathrm{H}, \mathrm{C} 7 \mathrm{H}\right), 5.36$ $\left(2 \mathrm{H}, \mathrm{d},{ }^{4} \mathrm{~J} 1.8, \mathrm{C} 4 \mathrm{H}, \mathrm{C} 5 \mathrm{H}\right), 3.38\left(6 \mathrm{H}, \mathrm{s}, 2 \times \mathrm{OCH}_{3}\right), 3.00(12 \mathrm{H}, \mathrm{s}$, $\left.2 \times \mathrm{N}\left(\mathrm{CH}_{3}\right)_{2}\right) ;{ }^{13} \mathrm{C} \mathrm{NMR}\left(125 \mathrm{MHz}, \mathrm{CDCl}_{3}\right): \delta=161.3(\mathrm{C} 1, \mathrm{C} 8)$, 155.4 (C3, C6), 154.6, 144.9 (C4a, C5a), 142.2 (C9), 139.2, 131.9, 130.6, 128.1, 126.7, 126.3, 126.0, 109.8 (C8a, C9a), 93.3 $(\mathrm{C} 2, \mathrm{C} 7), 89.5(\mathrm{C} 4, \mathrm{C} 5), 56.1\left(\mathrm{OCH}_{3}\right), 40.3\left(\mathrm{~N}\left(\mathrm{CH}_{3}\right)_{2}\right)$; ESI-MS: $\mathrm{m} / \mathrm{z}$ calcd. for $\mathrm{C}_{31} \mathrm{H}_{32} \mathrm{~N}_{3} \mathrm{O}_{2}{ }^{+} 478.2489$ found $478.2495\left[\mathrm{M}^{+}\right]$. Luminescence spectroscopy (in $\mathrm{MeCN}$ ): $\lambda_{\text {abs }}$ : $498 \mathrm{~nm}$; $\varepsilon_{\mathrm{abs}}$ : $4.0 \cdot 10^{4} \mathrm{~L} \cdot \mathrm{cm} \cdot \mathrm{mol}^{-1} ; \lambda_{\text {em }}($ exc 488$): 540 \mathrm{~nm}$; Stokes shift: $42 \mathrm{~nm}$; $\mathrm{E}_{0,0}: 2.40 \mathrm{eV} ;\left\langle\tau_{\mathrm{F}}>: 4.4 \mathrm{~ns}\right.$; Cyclic voltammetry (vs SCE): $\mathrm{E}_{1 / 2}\left(\mathrm{P}^{*} / \mathrm{P}^{-}\right):+1.21 \mathrm{~V}, \mathrm{E}_{1 / 2}\left(\mathrm{P} / \mathrm{P}^{-}\right):-1.19 \mathrm{~V}$.

\subsubsection{1,8-Dimethoxy-10-methyl-9-phenylacridinium bromide salt $(\mathbf{4 d})$}

The compound was prepared according to the general procedure $\mathrm{A}$ and $\mathrm{B}$ using 3-methoxy- $\mathrm{N}$-(3-methoxyphenyl)- $\mathrm{N}$ methylaniline (1d) (38.9 $\mathrm{mg}, 160 \mu \mathrm{mol})$ and methyl benzoate $(13.6 \mathrm{mg}, 100 \mu \mathrm{mol})$ and was stirred $12 \mathrm{~h}$ at RT. Purification gave a brown red solid $(19.9 \mathrm{mg}, 49 \%$, HPLC purity: 98.6\%; decomp. at $\left.150{ }^{\circ} \mathrm{C}\right): \mathrm{R}_{f} 0.12\left(\mathrm{CH}_{2} \mathrm{Cl}_{2}: \mathrm{MeOH} 10: 1\right)$; $v_{\max }$ (neat): $3411 \mathrm{w}, 1606 \mathrm{~m}, 1504 \mathrm{~m}, 1465 \mathrm{~m}, 1345 \mathrm{~m}, 1260 \mathrm{~s}$, $1168 \mathrm{~m}, 1072 \mathrm{~m}, 926 \mathrm{w}, 816 \mathrm{~m}, 729 \mathrm{~s}, 699 \mathrm{~s}, 632 \mathrm{~m} ;{ }^{1} \mathrm{H}$ NMR $\left(500 \mathrm{MHz}, \mathrm{CDCl}_{3}\right): \delta=8.24-8.28(2 \mathrm{H}, \mathrm{m}, \mathrm{C} 4 H, \mathrm{C} 5 H), 8.26-$ $8.30(2 \mathrm{H}, \mathrm{m}, \mathrm{C} 3 H, \mathrm{C} 6 H), 7.42-7.46\left(3 \mathrm{H}, \mathrm{m}, \mathrm{C} 3{ }^{\prime} H, \mathrm{C} 4{ }^{\prime} H, \mathrm{C} 5 ' H\right)$, 7.15-7.17 (2H, m, C2'H, C6'H), $7.01\left(2 \mathrm{H}, \mathrm{dd},{ }^{3} J\right.$ 7.2, ${ }^{4} J 1.2$, $\mathrm{C} 2 \mathrm{H}, \mathrm{C} 7 \mathrm{H}), 5.02\left(3 \mathrm{H}, \mathrm{s}, \mathrm{NCH}_{3}\right), 3.48\left(6 \mathrm{H}, \mathrm{s}, 2 \times \mathrm{OCH}_{3}\right) ;{ }^{13} \mathrm{C}$ NMR (125 MHz, $\left.\mathrm{CDCl}_{3}\right): \delta=161.1(C 9), 160.1(C 1, C 8), 142.3$ (C4a, C10a), $141.4\left(C 1^{\prime}\right), 140.8$ (C3, C6), $127.1\left(C 4^{\prime}\right), 126.8$ (C3', C5 '), 125.6 (C2', C6'), $119.1(C 8 a, C 9 a), 110.2(C 4, C 5)$, $106.8(C 2, C 7), 56.6\left(2 \times \mathrm{OCH}_{3}\right), 42.3\left(\mathrm{NCH}_{3}\right)$; ESI-MS: m/z calcd. for $\mathrm{C}_{22} \mathrm{H}_{20} \mathrm{NO}_{2}{ }^{+} 330.1489$ found $330.1494 \quad\left[\mathrm{M}^{+}\right]$. Luminescence spectroscopy (in MeCN): $\lambda_{\text {abs } 1}: 497 \mathrm{~nm} ; \lambda_{\text {abs } 2}: 403$ 
$\mathrm{nm} ; \varepsilon_{\mathrm{absl}}: 4.4 \cdot 10^{3} \mathrm{~L} \cdot \mathrm{cm} \cdot \mathrm{mol}^{-1} ; \varepsilon_{\text {abs } 2}: 6.7 \cdot 10^{3} \mathrm{~L} \cdot \mathrm{cm} \cdot \mathrm{mol}^{-1}, \lambda_{\text {em }}($ exc 487): $576 \mathrm{~nm} ; \lambda_{\text {em }}(\operatorname{exc} 393): 586 \mathrm{~nm}$; Stokes shift: $79 \mathrm{~nm} ; \mathrm{E}_{0,0}$ : $2.33 \mathrm{eV} ;\left\langle\tau_{\mathrm{F}}>: 2.7 \mathrm{~ns} ;\right.$ Cyclic voltammetry (vs $\left.\mathrm{SCE}\right): \mathrm{E}_{1 / 2}\left(\mathrm{P}^{*} / \mathrm{P}^{-}\right)$: $+1.81 \mathrm{~V}, \mathrm{E}_{1 / 2}\left(\mathrm{P} / \mathrm{P}^{-}\right):-0.52 \mathrm{~V}$.

\subsubsection{1,8-Dimethoxy-10-methyl-9-phenyl-9,10-} dihydroacridin-9-ol (4e)

The compound was prepared according to the general procedure A using using 3-methoxy- $N$-(3-methoxyphenyl)- $N$ methylaniline (1d) $(38.9 \mathrm{mg}, 160 \mu \mathrm{mol})$. To the metalated aniline in $n$ hexane $(160 \mu \mathrm{mol})$ at $-20{ }^{\circ} \mathrm{C}$ was added a solution of methyl benzoate $(13.6 \mathrm{mg}, 100 \mu \mathrm{mol})$ in anhydrous THF $(0.60 \mathrm{~mL})$ and the reaction mixture was allowed to warm to RT over $12 \mathrm{~h}$. Aqueous saturated $\mathrm{NH}_{4} \mathrm{Cl}(1.00 \mathrm{~mL})$ was added, followed by water $(20 \mathrm{~mL})$ and the mixture was extracted by $\mathrm{CH}_{2} \mathrm{Cl}_{2}(4 \mathrm{x} 10$ $\mathrm{mL}$ ). The combined organic layer was dried over $\mathrm{Na}_{2} \mathrm{SO}_{4}$, filtered and concentrated in vacuo. Recrystallization from hexane:toluene (7.0 mL, 5:3) gave a dark grey solid $(19.9 \mathrm{mg}, 43 \%$, m.p. $171.5-$ $173.9{ }^{\circ} \mathrm{C}$ ): $v_{\max }$ (neat): $3514 \mathrm{w}, 1596 \mathrm{~s}, 1470 \mathrm{~s}, 1374 \mathrm{w}, 1251 \mathrm{~m}$, $1171 \mathrm{w}, 1081 \mathrm{~s}, 1020 \mathrm{w}, 908 \mathrm{w}, 773 \mathrm{~s}, 725 \mathrm{~s}, 631 \mathrm{~m} ;{ }^{1} \mathrm{H}$ NMR $\left(500 \mathrm{MHz}, \mathrm{CDCl}_{3}\right): \delta=7.33-7.35\left(2 \mathrm{H}, \mathrm{m}, \mathrm{C} 2^{\prime} H, \mathrm{C}^{\prime} H\right), 7.21$ $\left(2 \mathrm{H}, \mathrm{t},{ }^{3} J 8.3, \mathrm{C} 3 H, \mathrm{C} 6 H\right), 7.13-7.16\left(2 \mathrm{H}, \mathrm{m}, \mathrm{C} 3{ }^{\prime} H, \mathrm{C}{ }^{\prime} H\right), 7.01-$ $7.04\left(1 \mathrm{H}, \mathrm{C} 4{ }^{\prime} H\right), 6.70\left(2 \mathrm{H}, \mathrm{dd},{ }^{3} J 8.4,{ }^{4} J 0.5, \mathrm{C} 4 H, \mathrm{C} 5 H\right), 6.44$ $\left(2 \mathrm{H}, \mathrm{dd},{ }^{3} J 8.2,{ }^{4} J 0.6, \mathrm{C} 2 \mathrm{H}, \mathrm{C} 7 \mathrm{H}\right), 5.17(1 \mathrm{H}, \mathrm{s}, \mathrm{OH}), 3.53(3 \mathrm{H}, \mathrm{s}$, $\left.\mathrm{NCH}_{3}\right), 3.51\left(6 \mathrm{H}, \mathrm{s}, 2 \times \mathrm{OCH}_{3}\right) ;{ }^{13} \mathrm{C}$ NMR $\left(125 \mathrm{MHz}, \mathrm{CDCl}_{3}\right)$ : $\delta=158.3(C 1, C 8), 150.8\left(C 1^{\prime}\right), 139.4(C 4 a, C 10 a), 128.8(C 3$, C6), 126.1 (C3', C5 '), 125.9 (C2', C6'), 125.0 (C4'), 117.0 (C8a, $C 9 a), 106.4$ (C4,C5), $105.0(C 2, C 7), 72.6(C 9), 55.9(2 \mathrm{x}$ $\left.\mathrm{OCH}_{3}\right), 35.2\left(\mathrm{NCH}_{3}\right)$. ESI-MS: m/z calcd. for $\mathrm{C}_{22} \mathrm{H}_{21} \mathrm{NNaO}_{3}{ }^{+}$ 370.1419 found $370.1418\left[\mathrm{MNa}^{+}\right]$.

\subsubsection{9-(4-Fluorophenyl)-1,8-dimethoxy-10- methylacridinium bromide salt (4f)}

The compound was prepared according to the general procedure A and B using 3-methoxy- $N$-(3-methoxyphenyl)- $N$ methylaniline (1d) $(38.9 \mathrm{mg}, 160 \mu \mathrm{mol})$ and methyl 4fluorobenzoate $(15.4 \mathrm{mg}, 100 \mu \mathrm{mol})$ and was stirred $12 \mathrm{~h}$ at RT. Purification gave a brown red solid $(17.5 \mathrm{mg}, 41 \%$, HPLC purity: 96.1\%; decomp. at $\left.130{ }^{\circ} \mathrm{C}\right): \mathrm{R}_{f} 0.13\left(\mathrm{CH}_{2} \mathrm{Cl}_{2}: \mathrm{MeOH} 10: 1\right)$; $v_{\max }$ (neat): $3379 \mathrm{w}, 1606 \mathrm{~m}, 1579 \mathrm{~m}, 1508 \mathrm{~s}, 1462 \mathrm{~s}, 1348 \mathrm{~m}, 1259 \mathrm{~s}$, $1219 \mathrm{~m}, 1167 \mathrm{~m}, 1072 \mathrm{~m}, 1025 \mathrm{w}, 919 \mathrm{~m}, 833 \mathrm{~m}, 816 \mathrm{~m}, 770 \mathrm{~m}$, $723 \mathrm{~m}, 635 \mathrm{~s} ;{ }^{1} \mathrm{H}$ NMR $\left(500 \mathrm{MHz}, \mathrm{CDCl}_{3}\right): \delta=8.27-8.29(4 \mathrm{H}, \mathrm{m}$, $\mathrm{C} 3 H, \mathrm{C} 4 H, \mathrm{C} 5 H, \mathrm{C} 6 H), 7.16-7.17$ (4H, m, C2' $H, \mathrm{C}^{\prime} H, \mathrm{C} 5 ' H$, C6'H), $7.04\left(2 \mathrm{H}, \mathrm{dd},{ }^{3} J 5.9,{ }^{4} J 2.9, \mathrm{C} 2 \mathrm{H}, \mathrm{C} 7 \mathrm{H}\right), 5.03(3 \mathrm{H}, \mathrm{s}$, $\left.\mathrm{NCH}_{3}\right), 3.54\left(6 \mathrm{H}, \mathrm{s}, 2 \times \mathrm{OCH}_{3}\right) ;{ }^{13} \mathrm{C} \mathrm{NMR}\left(125 \mathrm{MHz}, \mathrm{CDCl}_{3}\right)$ : $\delta=162.0\left({ }^{1} J_{C F} 247 \mathrm{~Hz}, C \mathrm{~F}\right), 159.9$ (C9), 159.8 (C1, C8), 142.3 $(C 4 a, C 10 a), 140.8(C 3, C 6), 137.3\left({ }^{4} J_{C F} 3.6 \mathrm{~Hz}, C 1\right), 127.6$ $\left({ }^{3} \mathrm{~J}_{C F} 8.0 \mathrm{~Hz}, C 2\right.$ ', C6 $), 119.2(C 8 a, C 9 a), 114.0\left({ }^{2} J_{C F} 21.8 \mathrm{~Hz}\right.$, $C 3^{\prime}, C 5$ '), 110.5 (C4,C5), 106.9 (C2, C7), 56.7 (2 x OCH $), 42.5$ $\left(\mathrm{NCH}_{3}\right) ;{ }^{19} \mathrm{~F}$ NMR $\left(235 \mathrm{MHz}, \mathrm{CDCl}_{3}\right)$ : 114.7; ESI-MS: m/z calcd. for $\mathrm{C}_{22} \mathrm{H}_{19} \mathrm{FNO}_{2}{ }^{+} 348.1394$ found $348.1397 \quad\left[\mathrm{M}^{+}\right]$. Luminescence spectroscopy (in MeCN): $\lambda_{\text {abs } 1}: 497 \mathrm{~nm} ; \lambda_{\text {abs2 }}: 395$ $\mathrm{nm} ; \varepsilon_{\text {abs } 1}: 3.9 \cdot 10^{3} \mathrm{~L} \cdot \mathrm{cm} \cdot \mathrm{mol}^{-1} ; \varepsilon_{\text {abs } 2}: 6.3 \cdot 10^{3} \mathrm{~L} \cdot \mathrm{cm} \cdot \mathrm{mol}^{-1}, \lambda_{\mathrm{em}}(\mathrm{exc}$ 487): $579 \mathrm{~nm} ; \lambda_{\text {em }}(\operatorname{exc} 385): 581 \mathrm{~nm}$; Stokes shift: $82 \mathrm{~nm} ; \mathrm{E}_{0,0}$ : $2.31 \mathrm{eV} ;\left\langle\tau_{\mathrm{F}}>: 3.0 \mathrm{~ns} ;\right.$ Cyclic voltammetry (vs SCE): $\mathrm{E}_{1 / 2}\left(\mathrm{P}^{*} / \mathrm{P}^{-}\right)$: $+1.80 \mathrm{~V}, \mathrm{E}_{1 / 2}\left(\mathrm{P} / \mathrm{P}^{-}\right):-0.51 \mathrm{~V}$.

\subsubsection{1,8-Dimethoxy-9-(4-methoxyphenyl)-10- methylacridinium bromide salt (4g)}

The compound was prepared according to the general procedure A and B using 3-methoxy- $N$-(3-methoxyphenyl)- $N$ methylaniline (1d) $(38.9 \mathrm{mg}, 160 \mu \mathrm{mol})$ and methyl 4methoxybenzoate $(16.6 \mathrm{mg}, 100 \mu \mathrm{mol})$ and was stirred $12 \mathrm{~h}$ at RT. Purification gave a brown red solid (17.2 mg, 39\%, HPLC purity: $96.3 \%$; decomp. at $\left.130{ }^{\circ} \mathrm{C}\right): \mathrm{R}_{f} 0.15\left(\mathrm{CH}_{2} \mathrm{Cl}_{2}: \mathrm{MeOH}\right.$ $10: 1)$; $v_{\max }$ (neat): 3375w, 2993w, $1606 \mathrm{~m}, 1577 \mathrm{~m}, 1509 \mathrm{~m}$,
$1461 \mathrm{~m}, 1346 \mathrm{~m}, 1240 \mathrm{~s}, 1165 \mathrm{~s}, 1032 \mathrm{~m}, 814 \mathrm{~m}, 765 \mathrm{~s}, 734 \mathrm{w}, 647 \mathrm{~m}$, $635 \mathrm{w} ;{ }^{1} \mathrm{H}$ NMR $\left(500 \mathrm{MHz}, \mathrm{CDCl}_{3}\right): \delta=8.22-8.28(4 \mathrm{H}, \mathrm{m}, \mathrm{C} 3 H$, $\mathrm{C} 4 H, \mathrm{C} 5 H, \mathrm{C} 6 H), 7.06-7.09\left(2 \mathrm{H}, \mathrm{m}, \mathrm{C} 2^{\prime} H, \mathrm{C} 6{ }^{\prime} H\right), 7.02(2 \mathrm{H}, \mathrm{dd}$, ${ }^{3} J$ 7.6, $\left.{ }^{4} J 0.9, \mathrm{C} 2 H, \mathrm{C} 7 H\right), 6.98-7.00\left(2 \mathrm{H}, \mathrm{m}, \mathrm{C} 3{ }^{\prime} H, \mathrm{C} 5{ }^{\prime} H\right), 5.00$ $\left(3 \mathrm{H}, \mathrm{s}, \mathrm{NCH}_{3}\right), 3.93\left(3 \mathrm{H}, \mathrm{s}, \mathrm{OCH}_{3}\right), 3.55(6 \mathrm{H}, \mathrm{s}, 2 \mathrm{x} \mathrm{OCH}) ;{ }^{13} \mathrm{C}$ NMR (125 MHz, $\left.\mathrm{CDCl}_{3}\right): \delta=161.6(C 9), 160.2(C 1, C 8), 158.9$ (C4'), 142.3 (C4a, C10a), 140.6 (C3, C6), 133.7 (C1'), 127.2 (C2', C6'), 119.5 (C8a,C9a), $\left.112.4 C 3^{\prime}, C 5^{\prime}\right), 110.1(C 4, C 5)$, $106.8(\mathrm{C} 2, \mathrm{C} 7), 56.8\left(2 \times \mathrm{OCH}_{3}\right), 55.5\left(\mathrm{OCH}_{3}\right), 42.3\left(\mathrm{NCH}_{3}\right)$; ESI-MS: $\mathrm{m} / \mathrm{z}$ calcd. for $\mathrm{C}_{23} \mathrm{H}_{22} \mathrm{NO}_{3}{ }^{+} 360.1594$ found 360.1595 $\left[\mathrm{M}^{+}\right]$. Luminescence spectroscopy (in MeCN): $\lambda_{\text {abs1 }}: 494 \mathrm{~nm}$; $\lambda_{\text {abs } 2}: 399 \mathrm{~nm} ; \varepsilon_{\text {abs } 1}: 4.5 \cdot 10^{3} \mathrm{~L} \cdot \mathrm{cm} \cdot \mathrm{mol}^{-1} ; \varepsilon_{\text {abs } 2}: 7.0 \cdot 10^{3} \mathrm{~L} \cdot \mathrm{cm} \cdot \mathrm{mol}^{-}$ $1, \lambda_{\text {em }}$ (exc 485): $567 \mathrm{~nm}$; $\lambda_{\text {em }}$ (exc 389): $569 \mathrm{~nm}$; Stokes shift: $72 \mathrm{~nm} ; \quad \mathrm{E}_{0,0}: 2.30 \mathrm{eV} ;\left\langle\tau_{\mathrm{F}}>: 5.9 \mathrm{~ns} ;\right.$ Cyclic voltammetry (vs $\mathrm{SCE}): \mathrm{E}_{1 / 2}\left(\mathrm{P}^{*} / \mathrm{P}^{-}\right):+1.68 \mathrm{~V}, \mathrm{E}_{1 / 2}\left(\mathrm{P} / \mathrm{P}^{-}\right):-0.62 \mathrm{~V}$.

\subsubsection{1,8-Dimethoxy-10-methyl-9-(naphthalen-1- yl)acridinium bromide salt (4h)}

The compound was prepared according to the general procedure $\mathrm{A}$ and $\mathrm{B}$ using 3-methoxy- $\mathrm{N}$-(3-methoxyphenyl)- $\mathrm{N}$ methylaniline (1d) $(38.9 \mathrm{mg}, 160 \mu \mathrm{mol})$ and methyl 1naphthoate $(18.6 \mathrm{mg}, 100 \mu \mathrm{mol})$ and was stirred $12 \mathrm{~h}$ at RT. Purification gave a brown red solid $(26.0 \mathrm{mg}, 57 \%$, HPLC purity: 95.7\%; ${ }^{19}$ decomp. at $\left.120{ }^{\circ} \mathrm{C}\right): \mathrm{R}_{f} 0.01\left(\mathrm{CH}_{2} \mathrm{Cl}_{2}: \mathrm{MeOH} 10: 1\right)$; $v_{\max }$ (neat): $3462 \mathrm{w}, 3396 \mathrm{w}, 1608 \mathrm{~m}, 1577 \mathrm{~m}, 1503 \mathrm{~m}, 1458 \mathrm{~s}$, $1348 \mathrm{~m}, 1258 \mathrm{~s}, 1161 \mathrm{~m}, 1065 \mathrm{~s}, 1015 \mathrm{w}, 950 \mathrm{w}, 800 \mathrm{~m}, 762 \mathrm{~s}, 647 \mathrm{w}$; ${ }^{1} \mathrm{H}$ NMR $\left(500 \mathrm{MHz}, \mathrm{CDCl}_{3}\right): \delta=8.36-8.38(2 \mathrm{H}, \mathrm{m}, \mathrm{C} 3 \mathrm{H}, \mathrm{C} 6 H)$, 8.24-8.27 (2H, m, C4H, C5H), 7.97-7.98 (1H, m, C5'H), 7.91$7.93(1 \mathrm{H}, \mathrm{m}, \mathrm{C} 4 ' H), 7.49-7.52\left(2 \mathrm{H}, \mathrm{m}, \mathrm{C} 3{ }^{\prime} H, \mathrm{C} 6{ }^{\prime} H\right), 7.32(1 \mathrm{H}$, ddd, $\left.{ }^{3} J 8.2,7.5,{ }^{4} J 1.2, \mathrm{C} 7{ }^{\prime} H\right), 7.19\left(1 \mathrm{H}, \mathrm{dd},{ }^{3} J 8.2,{ }^{4} J 0.7, \mathrm{C} 8{ }^{\prime} H\right)$, $6.96\left(1 \mathrm{H}, \mathrm{dd},{ }^{3} J 7.0,{ }^{4} J 1.0, \mathrm{C} 2{ }^{\prime} H\right), 6.88\left(2 \mathrm{H}, \mathrm{d},{ }^{3} J 8.0, \mathrm{C} 2 \mathrm{H}\right.$, $\mathrm{C} 7 \mathrm{H}), 5.14\left(3 \mathrm{H}, \mathrm{s}, \mathrm{NCH}_{3}\right), 3.08\left(6 \mathrm{H}, \mathrm{s}, 2 \times \mathrm{OCH}_{3}\right) ;{ }^{13} \mathrm{C} \mathrm{NMR}$ $\left(125 \mathrm{MHz}, \mathrm{CDCl}_{3}\right): \delta=160.2(C 9), 159.7(C 1, C 8), 142.4(C 4 a$, C10a), 140.6 (C4, C5), 139.9 (C4a'), 132.1 (C1'), 131.9 (C8a'), 128.1 (C5'), 127.4 (C4'), $126.3\left(C 7^{\prime}\right), 125.8\left(C 3^{\prime}\right), 125.0\left(C 8^{\prime}\right)$, 124.9 (C6'), $121.8\left(C 2{ }^{\prime}\right), 120.0(C 8 a, C 9 a), 110.7$ (C3, C6), $106.8(C 2, C 7), 56.4\left(2 \times \mathrm{OCH}_{3}\right), 42.5\left(\mathrm{NCH}_{3}\right) ;$ ESI-MS: $\mathrm{m} / \mathrm{z}$ calcd. for $\mathrm{C}_{26} \mathrm{H}_{22} \mathrm{NO}_{2}{ }^{+} 380.1645$ found $380.1648 \quad\left[\mathrm{M}^{+}\right]$. Luminescence spectroscopy (in MeCN): $\boldsymbol{\lambda}_{\text {abs } 1}: 497 \mathrm{~nm} ; \boldsymbol{\lambda}_{\text {abs } 2}: 394$ $\mathrm{nm} ; \boldsymbol{\varepsilon}_{\mathrm{abs} 1}: 5.0 \cdot 10^{3} \mathrm{~L} \cdot \mathrm{cm} \cdot \mathrm{mol}^{-1} ; \boldsymbol{\varepsilon}_{\text {abs } 2}: 7.6 \cdot 10^{3} \mathrm{~L} \cdot \mathrm{cm} \cdot \mathrm{mol}^{-1}, \boldsymbol{\lambda}_{\text {em }}(\mathrm{exc}$ 487): $531 \mathrm{~nm} ; \lambda_{\text {em }}(\operatorname{exc} 384): 580 \mathrm{~nm}$; Stokes shift: $34 \mathrm{~nm} ; \mathrm{E}_{0,0}$ : $2.39 \mathrm{eV} ;\left\langle\tau_{\mathrm{F}}>: 4.1 \mathrm{~ns} ;\right.$ Cyclic voltammetry (vs SCE): $\mathrm{E}_{1 / 2}\left(\mathrm{P}^{*} / \mathrm{P}^{-}\right)$: $+1.88 \mathrm{~V}, \mathrm{E}_{1 / 2}\left(\mathrm{P} / \mathrm{P}^{-}\right):-0.51 \mathrm{~V}$.

\section{Acknowledgments}

We gratefully acknowledge the Swiss National Science Foundation (BSSGI0-155902/1), the University of Basel, the NCCR Molecular Systems Engineering for generous financial support and PD Dr. D. Häussinger for assistance with NMR spectroscopy. We thank Prof. O. S. Wenger, Prof. M. Mayor, Prof. E. C. Constable and Prof. C. E. Housecroft for the use of their equipment for acquiring photophysical and electrochemical data and Dr. C. Kerzig, Dr. X. Guo for helpful discussions.

\section{References and notes}

1. a) Teegardin, K.; Day, J. I.; Chan, J.; Weaver, J. Org. Process Res. Dev. 2016, 20, 1156-1163; Larsen, C. B.; Wenger, O. S. Chem. Eur. J. 2018, 24, 2039-2058; b) Early applications of Ru(bpy) ${ }_{3}{ }^{2+}$ as a visible light sensitizer by Sir Derek Barton and co-workers: Barton, D. R.; Csiba, M. A.; Jaszberenyi, J. Cs. Tetrahedron Letters 1994, 35, 2869-2872. c) Douglas, J. J.; Sevrin, M. J.; Stephenson, C. R. J. Org. Process Res. Dev. 2016, 20, 1134-1147; Alpers, D.; Gallhof, M.; Witt, J.; Hoffmann, F.; Brasholz, M. Angew.Chem. Int.Ed. 2017, 56, 1402-1406; Nicholls, T. P.; Leonori, D.; Bissember, A. C. Nat. Prod. Rep. 2016, 33, 12481254; Prier, C. K.; Rankic, D. A.; MacMillan, D. W. C. Chem. Rev. 2013, 113, 5322-5363. 
2. a) Marzo, L.; Pagire, S. K.; Reiser, O.; König, B. Angew. Chem. Int. Ed. 2018, doi: 10.1002/anie.201709766; Romero, N. A. Nicewicz, D. A. Chem. Rev. 2016, 116, 10075-10166; b) Metternich, J. B.; Gilmour, R. J. Am. Chem. Soc. 2016, 138, 1040-1045; c) Joshi-Pangu, A.; Lévesque, F.; Roth, H. G.; Oliver, S. F.; Campeau, L.-C.; Nicewicz, D. A.; DiRocco, D. A. J. Org. Chem. 2016, 81, 7244-7249;

3. a) Seminal acridinium synthesis by electrophilic aromatic substitution: Bernthsen, A.; Bender, F. Ber. Dtsch. Chem. Ges. 1883, 16, 1802-1819. b) Modern electrophilic aromatic substitution reactions: Dubrovskiy, A. V.; Larock, R. C. J. Org. Chem. 2012, 77, 11232-11256; J. Yanke, Z. Xiu, CN201410198453, 2014; see also Ref. 2c.

4. Link, A.; Fischer, C.; Sparr, C. Angew. Chem. Int. Ed. 2015, 54, 12163-12166; Link, A.; Fischer, C.; Sparr, C. Synthesis 2017, 49, $397-402$.

5. Fischer, C.; Sparr, C. Angew. Chem. Int. Ed. 2018, doi: 10.1002/ange.201711296.

6. Grimm, J. B.; Brown, T. A.; Tkachuk, A. N.; Lavis, L. D. ACS Cent. Sci. 2017, 3, 975-986.

7. Kamada, K.; Fuku-en, S.; Minamide, S.; Ohta, K.; Kishi, R.; Nakano, M.; Matsuzaki, H.; Okamoto, H.; Higashikawa, H.; Inoue, K.; Kojima, S.; Yamamoto, Y. J. Am. Chem. Soc. 2013 135, 232-241. By following the general $\mathrm{S}_{\mathrm{E}} A r-$ protocol described in Ref. $4 \mathrm{c}$, using benzoyl chloride (2.10 equiv.), triflic acid (1.00 equiv.) and amine $\boldsymbol{2 b}$ (1.00 equiv.) in 1,4-dioxane, 3,6-dimethoxy9,10-diphenylacridinium bromide salt was accessed as a single regioisomer. Luminescence spectroscopy (in $\mathrm{MeCN}$ ): $\lambda_{\text {abs }}: 400$ $\mathrm{nm} ; \varepsilon_{\max }: 2.1 \cdot 10^{4} \mathrm{~L} \cdot \mathrm{cm} \cdot \mathrm{mol}^{-1} ; \lambda_{\mathrm{em}}(\operatorname{exc} 390 \mathrm{~nm}): 496 \mathrm{~nm} ; \mathrm{E}_{0,0}: 2.73$ eV; CV (vs. SCE): $\mathrm{E}_{1 / 2}\left(\mathrm{P}^{*} / \mathrm{P}^{-}\right):+1.99 \mathrm{~V}, \mathrm{E}_{1 / 2}\left(\mathrm{P} / \mathrm{P}^{-}\right):-0.74 \mathrm{~V} ;<\tau_{\mathrm{F}}>$ : $9.1 \mathrm{~ns}$.

8. Delgado, I. H.; Pascal, S.; Wallabregue, A.; Duwald, R.; Besnard, C.; Guénée, L.; Nançoz, C.; Vauthey, E.; Tovar, R. C.; Lunkley, J. L.; Muller, G.; Lacour, J. Chem. Sci. 2016, 7, 4685-4693.

9. For safety reasons, $N$-bromosuccinimide is added portionwise.

10. Giumanini, A. G.; Chiavari, G.; Musiani, M. M.; Rossi, P. Synthesis 1980, 9, 743-746; Sprung, M. M. Chem. Rev. 1940, 26, 297-338.

11. a) Snieckus, V. Chem. Rev. 1990, 90, 879-933; Schlosser, M. Angew. Chem. Int. Ed. 2005, 44, 376-393. b) For a discussion of sequential metalations of planar substrates during electrophile additions, see: Leroux, F. R.; Mortier, J. Directed Metalation of Arenes with Organolithiums, Lithium Amides and Superbases in Arene Chemistry: Reaction Mechanisms and Methods for Aromatic Compounds (Ed.: Mortier, J.); Hoboken, NJ: John Wiley \& Sons Inc.; 2016; 743-776.

12. A similar transmetalation was described, using continuous flow: Ketels, M.; Konrad, D. B.; Karaghiosoff, K.; Trauner, D.; Knochel, P. Org. Lett. 2017, 19, 1666-1669.

13. Reactions performed with 1a $(160 \mu \mathrm{mol})$ and DoM-Reagent $(320 \mu \mathrm{mol})$ in $n$ hexane $(2.0 \mathrm{~mL})$ at $65^{\circ} \mathrm{C}$ for the indicated time t. Ester $2(100 \mu \mathrm{mol})$ in THF $(0.60 \mathrm{~mL})$ added at $-20{ }^{\circ} \mathrm{C}$ and warmed to the indicated temperature $T$ for $12 \mathrm{~h}$ followed by aqueous work-up $\left(8.8 \mathrm{molL}^{-1}, \mathrm{HBr}\right)$.

14. Fukuzumi, S.; Kotani, H.; Ohkubo, K.; Ogo, S.; Tkachenko, N V.; Lemmetyinen, H.; J. Am. Chem. Soc. 2004, 126, 1600-1601; Tsudaka, T.; Kotani, H.; Ohkubo, K.; Nakagawa, T.; Tkachenko, N. V.; Lemmetyinen, H.; Fukuzumi, S. Chem. Eur. J. 2017, 23, 1306-1317.

15. Fischer, C.; Sparr, C. Patent application filed EP 17/188,288.

16. (a) Miroshnikova, O. V.; Hudson, T. H.; Gerena, L. Kyle, D. E.; Lin, A. J. J. Med. Chem. 2007, 50, 889-896;

(b) Hameed P, S.; Patil, V.; Solapure, S.; Sharma, U.; Madhavapeddi, P.; Raichurkar, A.; Chinnapattu, M.; Manjrekar, P.; Shanbhag, G.; Puttur, J.; Shinde, V.; Menasinakai, S.; Rudrapatana, S.; Achar, V.; Awasthy, D.; Nandishaiah, R.; Humnabadkar, V.; Ghosh, A.; Narayan, C.; Ramya, V. K.; Kaur, P.; Sharma, S.; Werngren, J.; Hoffner, S.; Panduga, V.; Kumar, C. N. N.; Reddy, J.; Kumar Kn, M.; Ganguly, S.; Bharath, S.; Bheemarao, U.; Mukherjee, K.; Arora, U.; Gaonkar, S.; Coulson, M.; Waterson, D.; Sambandamurthy, V. K.; de Sousa, S. M. J. Med. Chem. 2014, 57, 4889-4905;

(c) Lu, H.; Geng, Z.; Li, J.; Zou, D.; Wu, Y.; Wu, Y. Org. Lett. 2016, 18, 2774-2776.

17. Uchiyama, M.; Matsumoto, Y.; Nakamura, S.; Ohwada, T.; Kobayashi, N.; Yamashita, N.; Matsumiya, A.; Sakamoto, T. J. Am. Chem. Soc. 2004, 126, 8755-8759.

18. Alongside the desired product, mono-coupled 5-methoxy- $N^{1}, N^{I}$ dimethyl- $N^{3}$-phenylbenzene-1,3-diamine $\quad(129 \mathrm{mg}, \quad 27 \%)$ was isolated as a dark oil. Analytical data is in agreement with the intermediate of $\mathbf{1 b}$

19. Aliquote was additionally purified for analytical purposes by reversed phase semi-prep HPLC. 